Article

\title{
Identification of Absorbed Constituents in the Rabbit Plasma and Cerebrospinal Fluid after Intranasal Administration of Asari Radix et Rhizoma by HS-SPME-GC-MS and HPLC-APCI-IT-TOF-MS
}

\section{Chen Li ${ }^{\dagger}$, Feng Xu ${ }^{\dagger}$, De-Mei Xie, Yu Jing, Ming-Ying Shang, Guang-Xue Liu, Xuan Wang and Shao-Qing Cai *}

State Key Laboratory of Natural and Biomimetic Drugs, School of Pharmaceutical Sciences, Peking University Health Science Center, No.38, Xueyuan Road, Beijing 100191, China; E-Mails: clearfont@163.com (C.L.); xufeng_pharm@163.com (F.X.); xlzh99@163.com (D.-M.X.); jing_jy@126.com (Y.J.); myshang@bjmu.edu.cn (M.-Y.S.); guangxl@bjmu.edu.cn (G.-X.L.); xuanwang6818@bjmu.edu.cn (X.W.)

$\dagger$ These authors contributed equally to this work.

* Author to whom correspondence should be addressed; E-Mail: sqcai@bjmu.edu.cn; Tel./Fax: +86-10-8280-1693.

Received: 28 January 2014; in revised form: 18 March 2014 / Accepted: 14 April 2014 / Published: 17 April 2014

\begin{abstract}
Traditional Chinese Medicine (TCM) nasal therapy has been utilized to treat numerous diseases for over two millennia. It has many advantages compared with other routes. In this article, headspace-solid phase microextraction-gas chromatography-mass spectrometry and high performance liquid chromatography-atmospheric pressure chemical ionization-ion trap-time of flight-multistage mass spectrometry were applied for the first time to analyze the absorbed constituents in rabbit plasma and cerebrospinal fluid (CSF) after intranasal administration of Asari Radix et Rhizoma (AR). In total, 47 absorbed AR constituents including 14 monoterpenes, 10 phenylpropanoids, four benzene derivatives, two alkanes, nine $N$-alkylamides and eight lignans were tentatively identified in the rabbit plasma and CSF. Thirty-three absorbed constituents are found to have different bioactivities related to the pharmacological actions of AR through bibliography data retrieval. These indicated that many types of constituents of TCM can be absorbed at the nasal cavity into both rabbit blood and CSF. This is the first study to explore the absorption of AR, and comprehensively analyze the absorbed constituents after intranasal administration of TCM.
\end{abstract}


These findings extend our understanding of the effective substances of AR, and inspire us to make a hypothesis on the mechanism of additive effect of multiple constituents of TCMs, which is very worthy of further investigation.

Keywords: nasal therapy; Asarum heterotropoides var. mandshuricum; intranasal administration; effective substance; cerebrospinal fluid; absorption; SPME; GC-MS; LC-MS; additive effect

\section{Introduction}

Traditional Chinese Medicine (TCM) nasal therapy (Biliao in Chinese) is used to treat local or systemic diseases through intranasal administration using TCMs in the form of powders or extracts. It has a long history of well over a thousand years since first recorded in Huangdi Neijing in the Warring State Period (403-221 B.C.), and is widely recorded in Chinese medical classics. It can be used to treat numerous maladies including internal, surgical, gynecological, pediatric and otolaryngologic diseases in TCM [1]. Intranasal administration has many advantages such as rapid onset of action, improved pharmacological effect, low side effect and good treatment compliance compared with oral and parenteral routes [2].

Several clinical researches regarding TCM nasal therapy were reported [3], and there are a few studies concerning the pharmacokinetics of absorbed constituents after intranasal administration of TCM preparations or single compound isolated from TCMs [4-6]. However, these reports mainly focused on determination of the contents of one or two absorbed constituents, and there is no report about the comprehensive analysis of absorbed constituents after intranasal administration of TCM. Besides, the techniques applied in previous researches (e.g., HPLC) lacked enough sensitivity to detect the trace constituents absorbed in the biological samples such as cerebrospinal fluid (CSF).

Asari Radix et Rhizoma (AR), one of the most frequently used TCMs in TCM nasal therapy, is recorded in the Chinese Pharmacopeia as the dried roots and rhizomes of Asarum heterotropoides Fr. Schmidt var. mandshuricum (Maxim.) Kitag., A. sieboldii Miq. var. seoulense Nakai and A. sieboldii Miq. Its frequency of utilization ranks first among prescriptions to treat brain diseases [7] and second in the recipes to treat migraine [8]. It is clinically used to alleviate pain and rhinitis, mainly to treat headache. It has been reported to possess analgesic, anti-inflammatory, sedative, antispasmodic, anti-allergic, cardiovascular, antitussive, hypothermal and anticonvulsant effects $[9,10]$. However, there is no report regarding the analysis of absorbed constituents of AR after intranasal administration.

In this article, techniques with high sensitivity namely headspace-solid phase microextraction-gas chromatography-mass spectrometry (HS-SPME-GC-MS) and high performance liquid chromatographyatmospheric pressure chemical ionization-ion trap-time of flight-multistage mass spectrometry (HPLCAPCI-IT-TOF-MS ${ }^{n}$ ) are employed to analyze the absorbed constituents in the plasma and CSF of rabbits after intranasal administration of AR comprehensively. HS-SPME-GC-MS is introduced to identify the volatile absorbed constituents and HPLC-APCI-IT-TOF-MS ${ }^{\mathrm{n}}$ is applied to identify the non-volatile absorbed constituents. Finally, bioactivities of the absorbed constituents related to the pharmacological effects of AR are summarized to give a better understanding of the effective 
substances of AR after intranasal administration. As far as we know, this is the first report to explore the absorption of AR, and comprehensively analyze the absorbed constituents after intranasal administration of a TCM.

\section{Results and Discussion}

\subsection{Identification of Absorbed Constituents in Rabbit Plasma and CSF by HS-SPME-GC-MS}

HS-SPME was chosen as the extraction mode because in this mode the SPME fiber can avoid the direct contact with biological samples, which can protect the fiber and prolong the fiber lifetime. Figures 1-4 show the total ion chromatograms of the analyzed samples. In total, 26 absorbed constituents containing 14 monoterpenes (G1-G13, G15), six phenylpropanoids (G16, G18, G21, G24-G26), four benzene derivatives (G14, G17, G20, G22) and two alkanes (G19, G23) were identified in the plasma and CSF from the AR EtOAc extract group compared with the blank group. The results are summarized in Table 1 and Figure 5.

Figure 1. Total ion chromatograms of plasma obtained from (a) blank group and (b) Asari Radix et Rhizoma (AR) EtOAc extract group using HS-SPME-GC-MS.
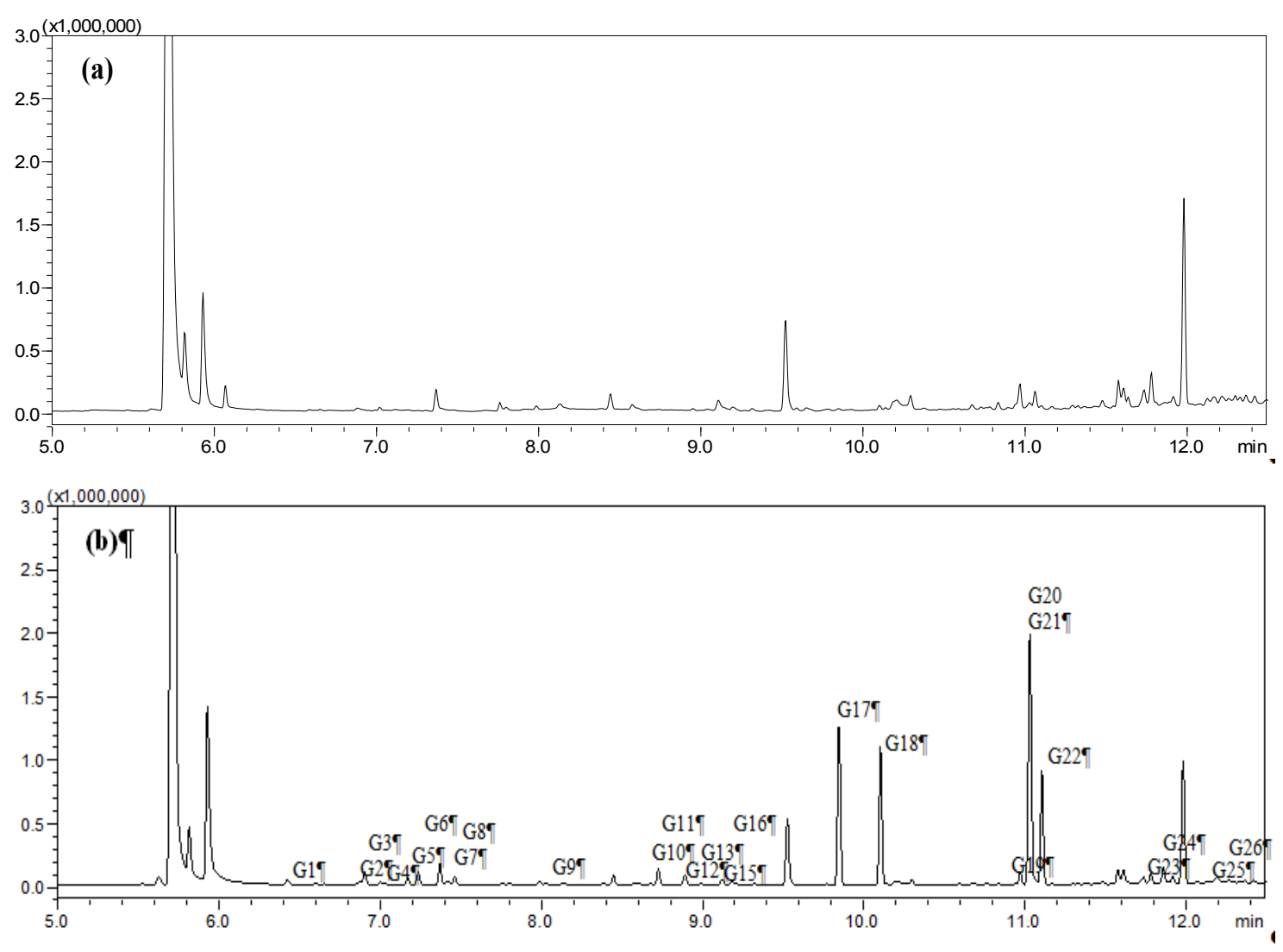
Figure 2. Total ion chromatograms of cerebrospinal fluid (CSF) obtained from (a) blank group and (b) AR EtOAc extract group using HS-SPME-GC-MS.
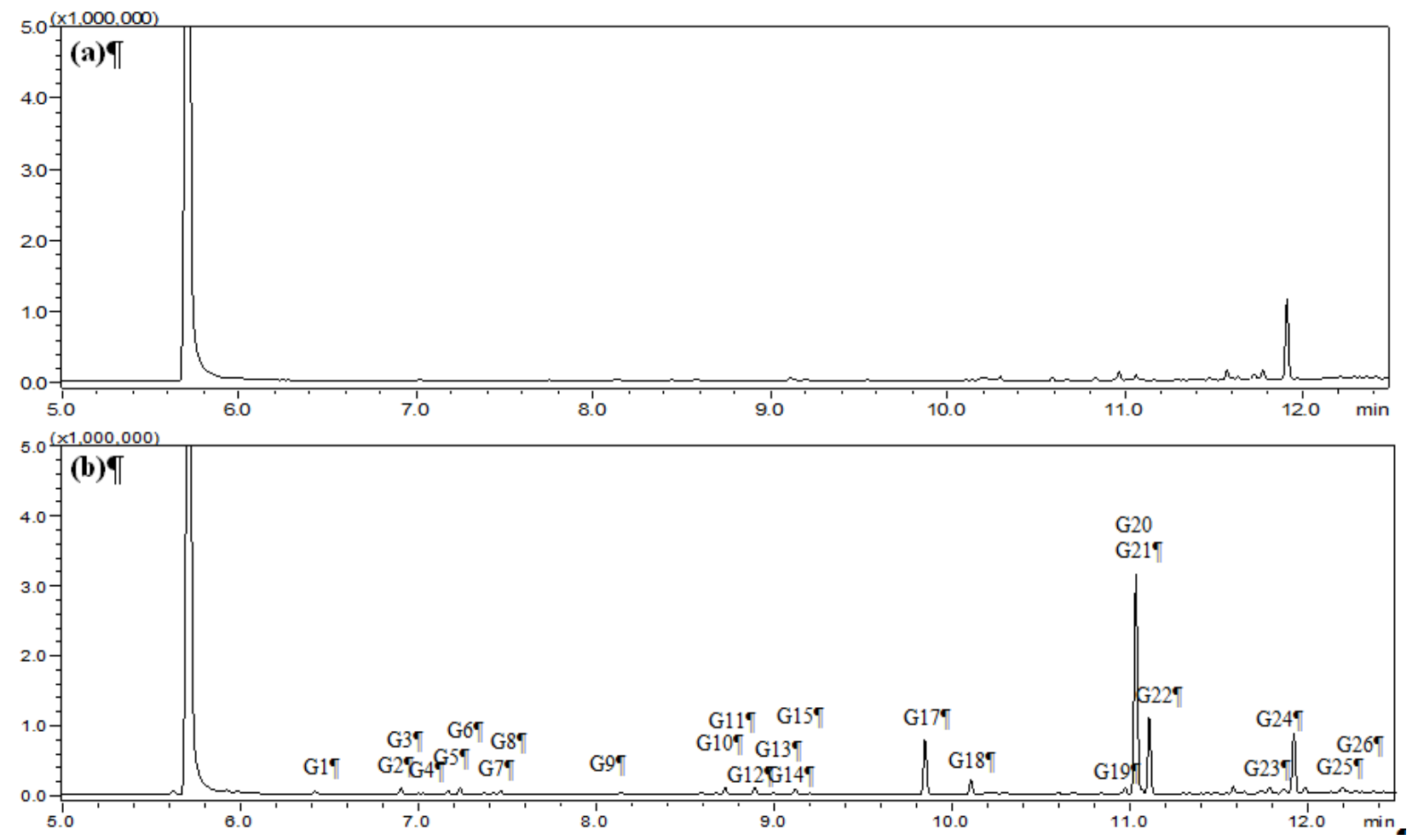

Figure 3. Total ion chromatograms of plasma obtained from (a) blank group and (c) Asari Radix et Rhizoma (AR) powder group using HS-SPME-GC-MS.
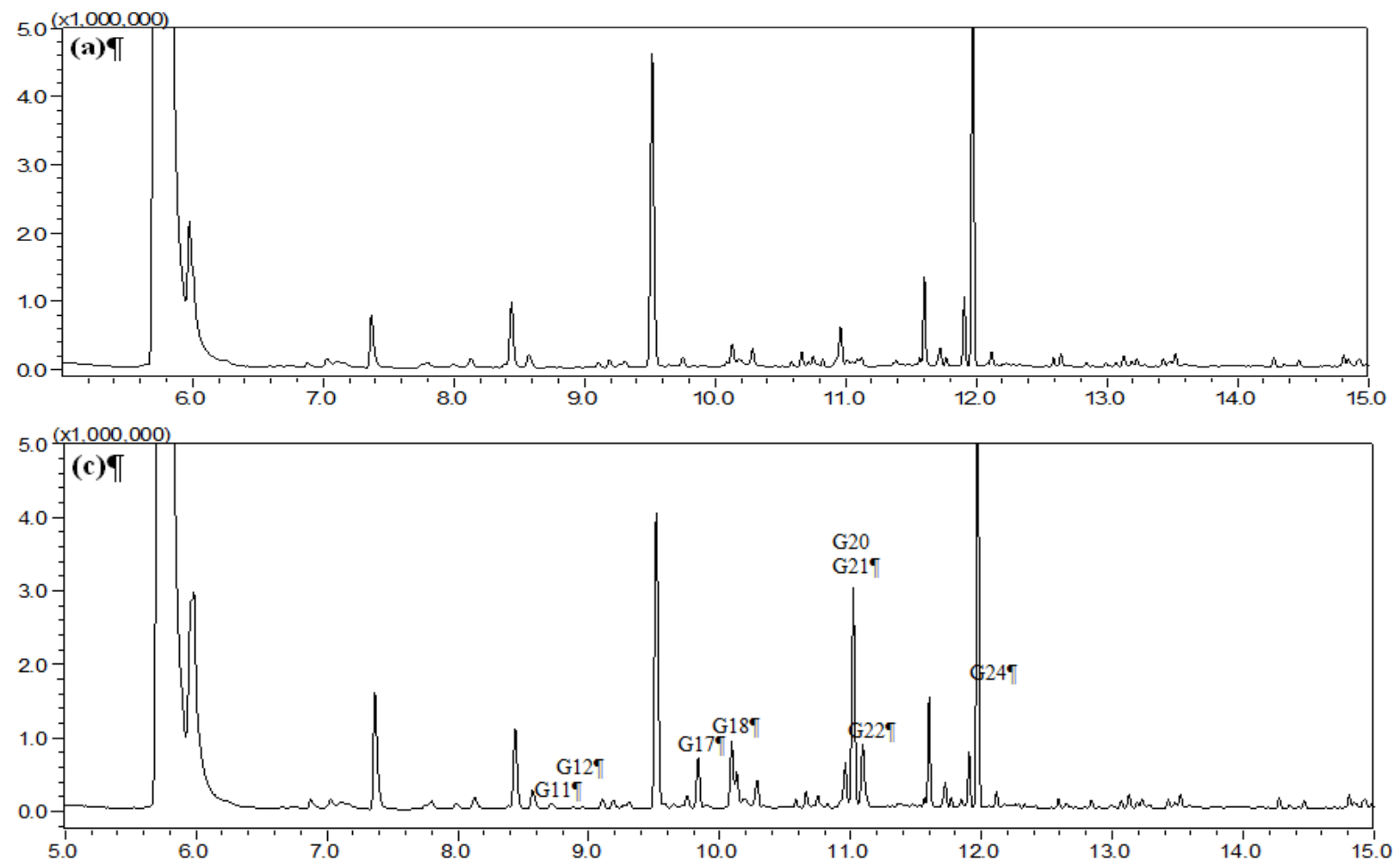
Figure 4. Total ion chromatograms of cerebrospinal fluid (CSF) obtained from (a) blank group and (c) Asari Radix et Rhizoma (AR) powder group using HS-SPME-GC-MS.
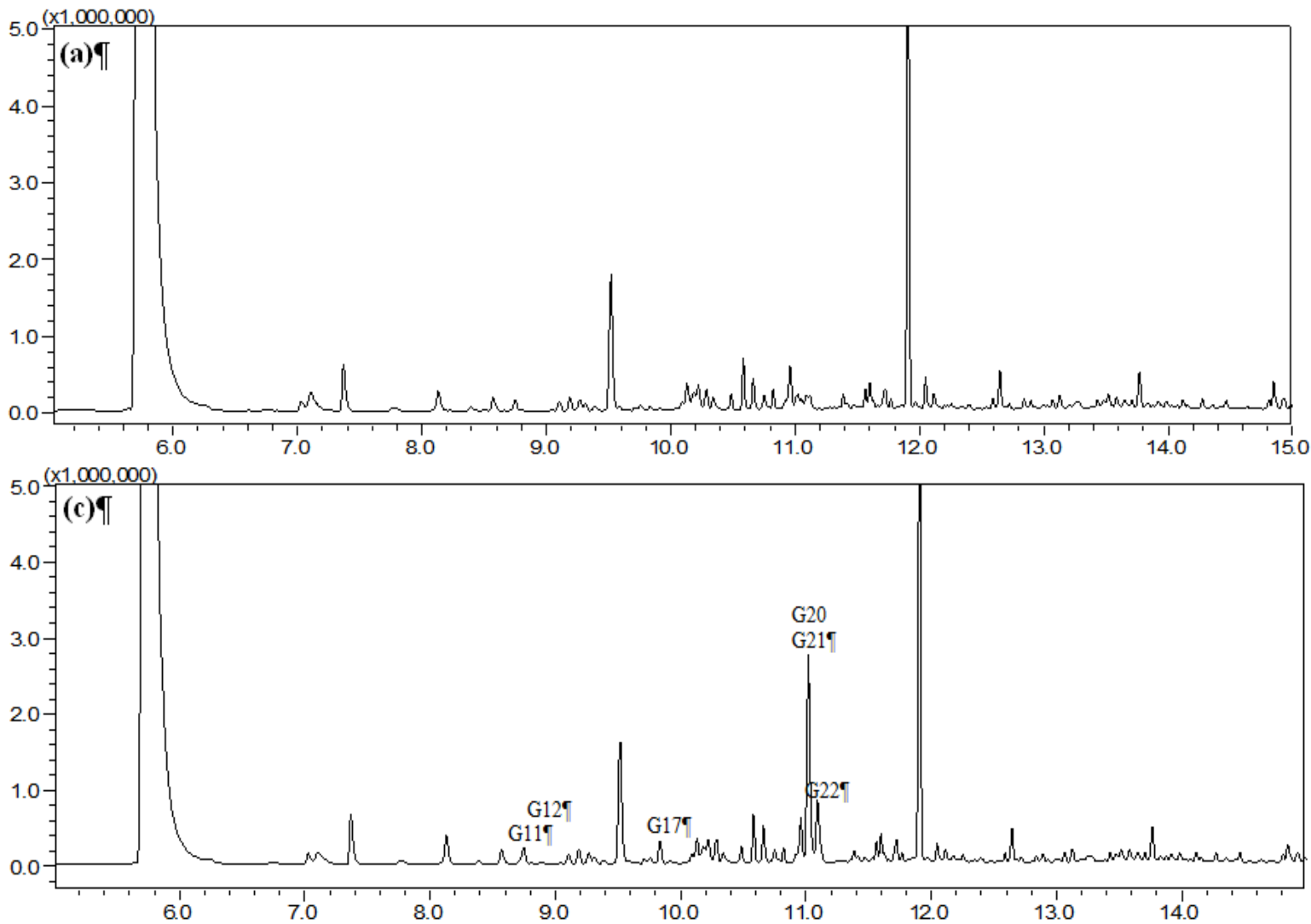

The AR EtOAc extract was reported to be the effective part of AR in analgesic activity [11], thus this extract was selected to study the absorbed constituents of AR after intranasal administration. Among the 26 absorbed constituents identified in the AR EtOAc extract group, 25 constituents were found in the plasma except $p$-cymen-8-ol, whereas 25 constituents were detected in the CSF aside from estragole.

Since AR is usually used in the form of powder in TCM nasal therapy clinically, the absorbed constituents after intranasal administration of AR powder were also studied and the absorbed constituents are supposed to be the effective substances of AR when it is used in nasal therapy. In the AR powder group, eight absorbed constituents (eucarvone, l-borneol, 3,5-dimethoxytoluene, safrole, 3,4,5-trimethoxytoluene, methyleugenol, 2,3,5-trimethoxytoluene and asaricin) were identified in the plasma and six of them (except safrole and asaricin) were detected in the CSF.

\subsection{Identification of Absorbed Constituents in Rabbit Plasma and CSF by HPLC-APCI-IT-TOF-MS ${ }^{n}$}

To reveal more constituents that could be absorbed into plasma and CSF, LCMS-IT-TOF was employed. Both APCI and ESI sources were tested in positive and negative ion modes. It was found that APCI gave stronger ion signals in positive ion mode. Hence APCI was selected as the ion source in the subsequent experiments monitored in the positive ion mode.

The fragmentation behaviors of four reference compounds (for their names see 2.2.1 below) in APCI-MS ${ }^{\mathrm{n}}$ were studied at first in order to facilitate the structure characterization of the absorbed 
constituents. Afterwards, the absorbed constituents were identified or tentatively characterized by the following procedures: (1) compare the base peak chromatograms (BPCs) of the experimental groups with that of the blank group to find characteristic peaks of them; (2) confirm the existence of them by comparing their characteristic extracted ion chromatograms (EICs) among 3 groups; (3) predict their formulae by high resolution MS data obtained by IT-TOF; (4) elucidate their structures by high resolution IT-TOF $\mathrm{MS}^{\mathrm{n}}$ data and by comparison with literature; (5) confirm their structures by comparison with the reference compounds as far as possible.

Table 1. The absorbed constituents in rabbit plasma and cerebrospinal fluid (CSF) from Asari Radix et Rhizoma (AR) EtOAc extract group (group b) and AR powder group (group c) identified by HS-SPME-GC-MS.

\begin{tabular}{|c|c|c|c|c|c|c|c|}
\hline \multirow{2}{*}{$\begin{array}{c}\text { Compound } \\
\text { No. }\end{array}$} & \multirow{2}{*}{$t_{R}(\min )$} & \multirow{2}{*}{$\mathrm{RI}^{\mathrm{b}}[12]$} & \multirow{2}{*}{ Constituents } & \multicolumn{2}{|c|}{ Group b } & \multicolumn{2}{|c|}{ Group c } \\
\hline & & & & Plasma & $\mathrm{CSF}$ & Plasma & $\mathrm{CSF}$ \\
\hline G1 & 6.423 & 944 & $\alpha$-Pinene & + & + & - & - \\
\hline G2 & 6.853 & 982 & Sabinene & + & + & - & - \\
\hline G3 & 6.902 & 988 & $\beta$-Pinene & + & + & - & - \\
\hline G4 & 7.000 & 993 & Myrcene & + & + & - & - \\
\hline G5 & 7.170 & 1012 & $\alpha$-Phellandrene & + & + & - & - \\
\hline G6 & 7.236 & 1018 & 3-Carene & + & + & - & - \\
\hline G7 & 7.421 & 1035 & Limonene & + & + & - & - \\
\hline G8 & 7.463 & 1040 & Eucalyptol & + & + & - & - \\
\hline G9 & 8.031 & 1096 & Terpinolene & + & + & - & - \\
\hline G10 & 8.675 & 1158 & Camphor & + & + & - & - \\
\hline G11 ${ }^{\mathrm{a}}$ & 8.724 & 1162 & Eucarvone & + & + & + & + \\
\hline $\mathrm{G} 12^{\mathrm{a}}$ & 8.890 & 1178 & l-Borneol & + & + & + & + \\
\hline G13 & 8.992 & 1188 & Terpinen-4-ol & + & + & - & - \\
\hline G14 & 9.035 & 1192 & $p$-Cymen-8-ol & - & + & - & - \\
\hline G15 & 9.118 & 1200 & $\alpha$-Terpineol & + & + & - & - \\
\hline G16 & 9.179 & 1206 & Estragole & + & - & - & - \\
\hline $\mathrm{G} 17^{\mathrm{a}}$ & 9.847 & 1275 & 3,5-Dimethoxytoluene & + & + & + & + \\
\hline G18 ${ }^{a}$ & 10.105 & 1300 & Safrole & + & + & + & - \\
\hline G19 & 10.971 & 1400 & Tetradecane & + & + & - & - \\
\hline $\mathrm{G} 20^{\mathrm{a}}, \mathrm{G} 21^{\mathrm{a}}$ & 11.030 & 1408 & 3,4,5-Trimethoxy-toluene/methyleugenol & + & + & + & + \\
\hline $\mathrm{G} 22^{\mathrm{a}}$ & 11.106 & 1417 & 2,3,5-Trimethoxytoluene & + & + & + & + \\
\hline G23 & 11.783 & 1501 & Pentadecane & + & + & - & - \\
\hline G24 & 11.860 & 1510 & Asaricin & + & + & + & - \\
\hline G25 & 12.191 & 1550 & 3,4-Methylenedioxy-propiophenone & + & + & - & - \\
\hline G26 & 12.268 & 1560 & Elemicin & + & + & - & - \\
\hline Sum & & & & 25 & 25 & 8 & 6 \\
\hline
\end{tabular}

${ }^{\mathrm{a}}$ Confirmed by comparison with reference compounds; ${ }^{\mathrm{b}}$ retention index; + detected; - not detected. 
Figure 5. Chemical structures of 26 absorbed constituents identified in rabbit plasma and CSF from AR EtOAc extract group and the AR powder group identified by HS-SPME-GC-MS.

\section{4 monoterpenes}

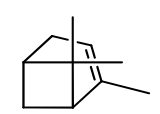

G1

$\alpha$-pinene

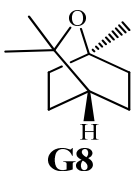

eucalyptol

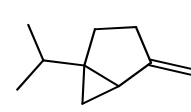

G2

sabinene

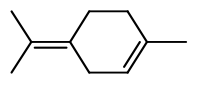

G9

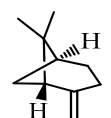

G3

$\beta$-pinene

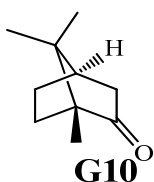

terpinolene<smiles>C=CC(=C)CCC=C(C)C</smiles>

G4

myrcene

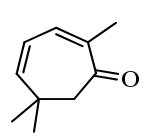

G11

eucarvone

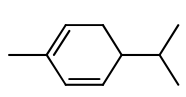

G5

$\alpha$-phellandrene

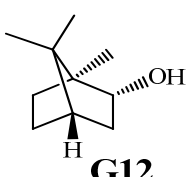

borneol

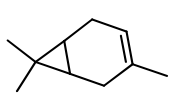

G6

3-carene

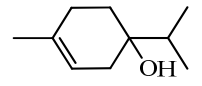

G13

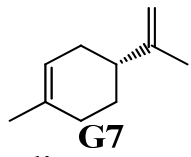

limonene

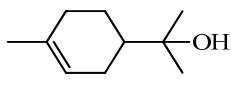

G15

$\alpha$-terpineol

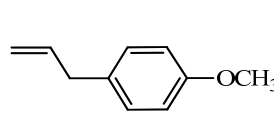

G16

estragole<smiles>C=CCc1ccc(OC)c(OC)c1</smiles>

G21

methyleugenol

6 phenylpropanoids

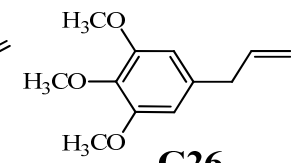

elemicin

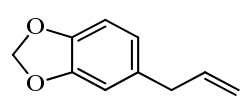

G18

safrole

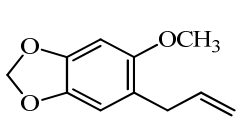

G24 asaricin

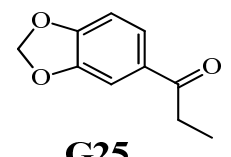

G25

3,4-methylenedioxypropiophenone

4 benzene derivatives

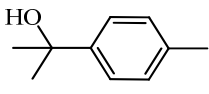

G14

p-cymen-8-ol

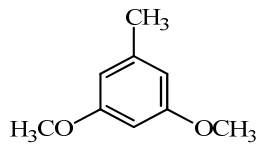

G17

3,5-dimethoxytoluene<smiles>COc1cc(C)c(OC)c(OC)c1</smiles>

G22 (=L7)

2,3,5-trimethoxytoluene

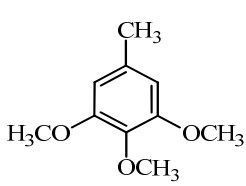

G20 (=L6)

3,4,5-trimethoxytoluene

\section{2 alkanes}
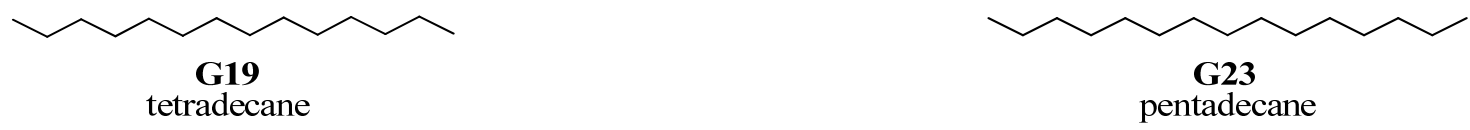

In total, 23 absorbed constituents including eight lignans (L1-L3, L5, L8, L10, L14, L15), nine $N$-alkylamides (L13, L16-L23), four phenylpropanoids (L4, L9, L11, L12) and two benzene derivatives $(\mathbf{L 6}$, L7) were identified in the plasma of the AR EtOAc extract group, but only five of them (L6, L7, L15-L17) were detected in the CSF. As for the AR powder group, only two compounds (L16, L17) were detected in the plasma and no compounds could be detected in the CSF. Among the above compounds, L4, L8, L12, L13, and L20-L23 were the compounds which did not reported in A. heterotropoides var. mandshuricum. The results are summarized in Table 2 and Figure 6. 
Table 2. The absorbed constituents in rabbit plasma and CSF from AR EtOAc extract group (group b) and AR powder group (group c) identified by HPLC-APCI-IT-TOF-MS ${ }^{\mathrm{n}}$.

\begin{tabular}{|c|c|c|c|c|c|c|c|c|c|c|c|c|}
\hline \multirow{2}{*}{ No. } & \multirow{2}{*}{$\begin{array}{c}t_{R} \\
(\min )\end{array}$} & \multirow{2}{*}{$\begin{array}{c}\text { Meas. } \\
\text { (Da) }\end{array}$} & \multirow{2}{*}{$\begin{array}{l}\text { Pred. } \\
\text { (Da) }\end{array}$} & \multirow{2}{*}{$\begin{array}{c}\text { Err. } \\
(\mathbf{p p m})\end{array}$} & \multirow{2}{*}{ DBE } & \multirow{2}{*}{ Formula } & \multirow{2}{*}{ Identification results } & \multirow{2}{*}{ Characteristic fragment ions } & \multicolumn{2}{|c|}{ Group b } & \multicolumn{2}{|c|}{ Group c } \\
\hline & & & & & & & & & $\mathbf{P}$ & $\mathbf{C}$ & $\mathbf{P}$ & $\mathbf{C}$ \\
\hline $\mathbf{L 1}$ & 11.486 & 359.1501 & 359.1489 & 3.34 & 10 & $\mathrm{C}_{20} \mathrm{H}_{22} \mathrm{O}_{6}$ & Epipinoresinol isomer & $\begin{array}{c}341.1380,323.1279,291.1026,271.1010 \\
259.0748,137.0618\end{array}$ & + & - & - & - \\
\hline $\mathbf{L 2}$ & 14.864 & 359.1489 & 359.1489 & 0 & 10 & $\mathrm{C}_{20} \mathrm{H}_{22} \mathrm{O}_{6}$ & Epipinoresinol isomer & $\begin{array}{c}341.1373,323.1263,291.1021,271.0964 \\
259.0781,137.0620\end{array}$ & + & - & - & - \\
\hline $\mathbf{L 3}$ & 22.813 & 357.1325 & 357.1333 & -2.24 & 11 & $\mathrm{C}_{20} \mathrm{H}_{20} \mathrm{O}_{6}$ & Xanthoxylol isomer & $\begin{array}{c}339.1218,321.1155,291.0999,289.0903 \\
269.0786,137.0464,135.0417\end{array}$ & + & - & - & - \\
\hline $\mathbf{L 4}$ & 26.497 & 211.0955 & 211.0965 & -4.74 & 5 & $\mathrm{C}_{11} \mathrm{H}_{14} \mathrm{O}_{4}$ & 3,4-Dimethoxybenzenepropionic acid & $\begin{array}{c}193.0846,178.0611,165.0904,161.0598 \\
133.0630\end{array}$ & + & - & - & - \\
\hline $\mathbf{L 5}^{\mathrm{a}}$ & 27.031 & 359.1489 & 359.1489 & 0 & 10 & $\mathrm{C}_{20} \mathrm{H}_{22} \mathrm{O}_{6}$ & Epipinoresinol & $\begin{array}{c}341.1372,323.1263,291.1021,271.0946 \\
259.0781,137.0620\end{array}$ & + & - & - & - \\
\hline $\mathbf{L 6}^{\mathrm{a}}$ & 31.577 & 183.1007 & 183.1016 & -4.92 & 4 & $\mathrm{C}_{10} \mathrm{H}_{14} \mathrm{O}_{3}$ & 3,4,5-Trimethoxytoluene & $168.0772,152.0825,151.0765$ & + & + & - & - \\
\hline $\mathbf{L} 7^{\text {a }}$ & 35.188 & 183.1007 & 183.1016 & -4.92 & 4 & $\mathrm{C}_{10} \mathrm{H}_{14} \mathrm{O}_{3}$ & 2,3,5-Trimethoxytoluene & $168.0772,152.0825,151.0723$ & + & + & - & - \\
\hline L8 & 36.811 & 387.1428 & 387.1438 & -2.58 & 11 & $\mathrm{C}_{21} \mathrm{H}_{22} \mathrm{O}_{7}$ & $(1 R, 2 S, 5 R, 6 R)-5^{\prime}-O-$ Methylpluviatilol & $\begin{array}{c}369.1354,351.1237,339.1198,319.0954 \\
299.0947,167.0723,135.0462\end{array}$ & + & - & - & - \\
\hline $\mathbf{L 9}^{\mathrm{a}}$ & 37.013 & 209.0800 & 209.0808 & -3.83 & 6 & $\mathrm{C}_{11} \mathrm{H}_{12} \mathrm{O}_{4}$ & Kakuol methyl ether & $191.0710,176.0459,161.0614,133.0621$ & + & - & - & - \\
\hline $\mathbf{L 1 0}^{\mathrm{a}}$ & 38.435 & 357.1333 & 357.1333 & 0 & 11 & $\mathrm{C}_{20} \mathrm{H}_{20} \mathrm{O}_{6}$ & Xanthoxylol & $\begin{array}{c}339.1222,321.1147,291.1048,289.0874 \\
269.0807,137.0545,135.0423\end{array}$ & + & - & - & - \\
\hline $\mathbf{L 1 1}^{\mathrm{a}}$ & 38.733 & 195.0644 & 195.0652 & -4.1 & 6 & $\mathrm{C}_{10} \mathrm{H}_{10} \mathrm{O}_{4}$ & Kakuol & $\begin{array}{c}177.0522,147.0418,139.0386,137.0179 \\
119.0478,109.0261\end{array}$ & + & - & - & - \\
\hline L12 & 39.104 & 197.0804 & 197.0808 & -2.03 & 5 & $\mathrm{C}_{10} \mathrm{H}_{12} \mathrm{O}_{4}$ & Hydroferulic acid & $\begin{array}{c}180.0751,179.0720,155.0732,151.0756 \\
133.0620,123.0894\end{array}$ & + & - & - & - \\
\hline L13 & 51.123 & 222.1842 & 222.1852 & -4.5 & 4 & $\mathrm{C}_{14} \mathrm{H}_{23} \mathrm{NO}$ & Spilanthol & $\begin{array}{c}167.1237,166.1329,152.0970,149.0985 \\
123.1139,121.1017,81.0807\end{array}$ & + & - & - & - \\
\hline L14 & 51.317 & 355.1191 & 355.1176 & 4.22 & 12 & $\mathrm{C}_{20} \mathrm{H}_{18} \mathrm{O}_{6}$ & $l$-Sesamin & $\begin{array}{c}337.1068,319.0966,289.0837,261.0917 \\
231.0791,203.0856,135.0418\end{array}$ & + & - & - & - \\
\hline
\end{tabular}


Table 2. Cont.

\begin{tabular}{|c|c|c|c|c|c|c|c|c|c|c|c|c|}
\hline \multirow{2}{*}{ No. } & \multirow{2}{*}{$\begin{array}{c}t_{R} \\
(\min )\end{array}$} & \multirow{2}{*}{$\begin{array}{l}\text { Meas. } \\
\text { (Da) }\end{array}$} & \multirow{2}{*}{$\begin{array}{l}\text { Pred. } \\
\text { (Da) }\end{array}$} & \multirow{2}{*}{$\begin{array}{l}\text { Err. } \\
(\text { ppm) }\end{array}$} & \multirow{2}{*}{ DBE } & \multirow{2}{*}{ Formula } & \multirow{2}{*}{ Identification results } & \multirow{2}{*}{ Characteristic fragment ions } & \multicolumn{2}{|c|}{ Group b } & \multicolumn{2}{|c|}{ Group c } \\
\hline & & & & & & & & & $\mathbf{P}$ & $\mathbf{C}$ & $\mathbf{P}$ & $\mathbf{C}$ \\
\hline $\mathbf{L 1 5}^{\text {a }}$ & 54.163 & 355.1178 & 355.1176 & 0.56 & 12 & $\mathrm{C}_{20} \mathrm{H}_{18} \mathrm{O}_{6}$ & $l$-asarinin & $\begin{array}{c}337.1075,319.0978,289.0875,261.0916 \\
231.0784,203.0850,135.0425\end{array}$ & + & + & - & - \\
\hline L16 & 56.775 & 248.2016 & 248.2009 & 2.82 & 5 & $\mathrm{C}_{16} \mathrm{H}_{25} \mathrm{NO}$ & $\begin{array}{l}N \text {-isobutyl-2E,4E, } 8 Z, 10 Z- \\
\quad \text { dodecatetraenamide }\end{array}$ & $\begin{array}{c}192.1362,175.1124,167.1304,166.1217 \\
149.1325,147.1173,142.1218,121.1007 \\
107.0847\end{array}$ & + & + & + & - \\
\hline $\mathbf{L 1 7}$ & 57.218 & 248.2005 & 248.2009 & -1.61 & 5 & $\mathrm{C}_{16} \mathrm{H}_{25} \mathrm{NO}$ & $\begin{array}{l}N \text {-isobutyl-2E,4E, } 8 Z, 10 E \text { - } \\
\text { dodecatetraenamide }\end{array}$ & $\begin{array}{c}192.1362,175.1124,167.1289,166.1232 \\
149.1325,147.1174,142.1206,121.0999 \\
107.0852\end{array}$ & + & + & + & - \\
\hline L18 & 58.080 & 248.1997 & 248.2009 & -4.83 & 5 & $\mathrm{C}_{16} \mathrm{H}_{25} \mathrm{NO}$ & $\begin{array}{c}N \text {-isobutyl-2,4,8,10-dodecatetraenamide } \\
\text { isomer }\end{array}$ & $\begin{array}{c}192.1452,175.1116,167.1284,166.1255 \\
149.1296,147.1160,142.1191,121.1002 \\
107.0820\end{array}$ & + & - & - & - \\
\hline L19 & 61.053 & 248.2004 & 248.2009 & -2.01 & 5 & $\mathrm{C}_{16} \mathrm{H}_{25} \mathrm{NO}$ & $\begin{array}{c}N \text {-isobutyl-2,4,8,10-dodecatetraenamide } \\
\text { isomer }\end{array}$ & $\begin{array}{c}175.1135,167.1339,149.1333,147.1138 \\
133.0672,121.1017,107.0813\end{array}$ & + & - & - & - \\
\hline $\mathbf{L 2 0}$ & 62.647 & 250.2150 & 250.2165 & -5.99 & 4 & $\mathrm{C}_{16} \mathrm{H}_{27} \mathrm{NO}$ & $N$-isobutyl-2,4,8-dodecatrienamide & $\begin{array}{c}194.1625,177.1164,167.1302,152.1070 \\
149.1398,109.0863,95.0789\end{array}$ & + & - & - & - \\
\hline $\mathbf{L 2 1}$ & 63.995 & 274.2164 & 274.2165 & -0.36 & 6 & $\mathrm{C}_{18} \mathrm{H}_{27} \mathrm{NO}$ & $\begin{array}{l}N \text {-isobutyl-2,4,8,10,12- } \\
\text { tetradecapentaenamide }\end{array}$ & $201.1332,175.1411,173.1332$ & + & - & - & - \\
\hline $\mathbf{L 2 2}$ & 66.168 & 276.2322 & 276.2322 & 0 & 5 & $\mathrm{C}_{18} \mathrm{H}_{29} \mathrm{NO}$ & $\begin{array}{l}N \text {-isobutyl-2,4,8,10- } \\
\text { tetradecatetraenamide }\end{array}$ & $\begin{array}{c}220.1623,203.1457,177.1611,175.1431 \\
167.1297,135.1181,133.0970\end{array}$ & + & - & - & - \\
\hline $\mathbf{L 2 3}$ & 66.563 & 252.2317 & 252.2322 & -1.98 & 3 & $\mathrm{C}_{16} \mathrm{H}_{29} \mathrm{NO}$ & $\mathrm{N}$-isobutyl-2,4-dodecadienamide & $196.1744,179.1256,154.1227,95.0480$ & + & - & - & - \\
\hline Sum & & & & & & & & & 23 & 5 & 2 & 0 \\
\hline
\end{tabular}

${ }^{a}$ Confirmed by comparison with reference compounds; DBE, double bond equivalent; + detected; - not detected; P plasma; C cerebrospinal fluid. 
Figure 6. Chemical structures of 23 absorbed constituents identified in rabbit plasma from AR EtOAc extract group identified by HPLC-APCI-IT-TOF-MS ${ }^{\mathrm{n}}$.

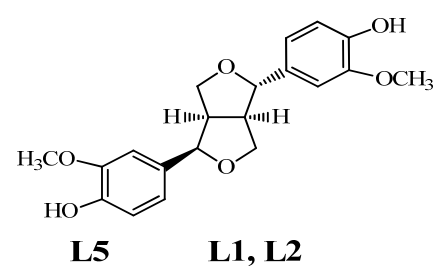

epipinoresinol epipinoresinol isomer

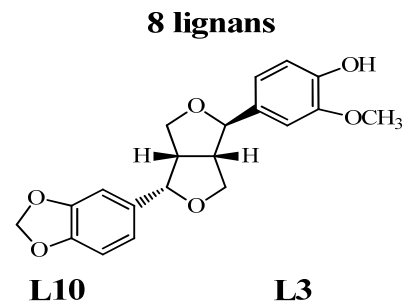

xanthoxylol xanthoxylol isomer

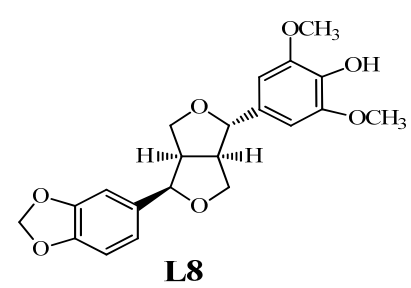

(1R,2S,5R,6R)-5'-O-methylpluviatilol

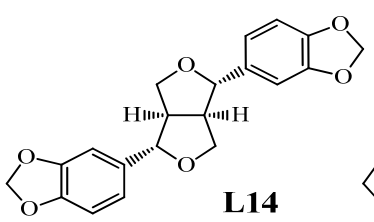

l-sesamin

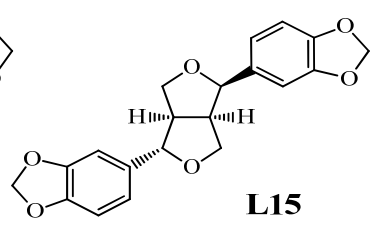

l-asarinin

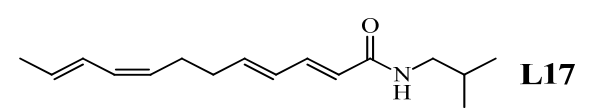

$N$-isobutyl-2E,4E,8Z,10E-dodecatetraenamide

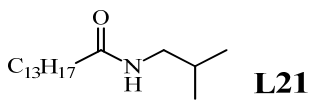

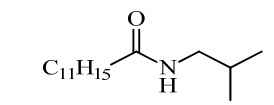

$N$-isobutyl-2,4,8,10dodecatetraenamide isomers

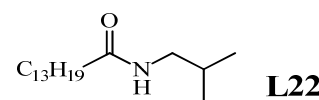

9 N-alkylamides

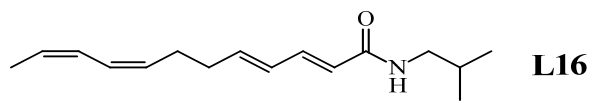

$N$-isobutyl-2E,4E,8Z,10Z-dodecatetraenamide

L18, L19

$N$-isobutyl-2,4,8-dodecatrienamide

$$
\mathrm{C}_{11} \mathrm{H}_{19} \overbrace{\mathrm{H}}^{\mathrm{O}} \overbrace{\mathrm{L23}}^{\mathrm{L}}
$$

$N$-isobutyl-2,4,8,10,12-tetradecapentaenamide $N$-isobutyl-2,4,8,10-tetradecatetraenamide $N$-isobutyl-2,4-dodecadienamide

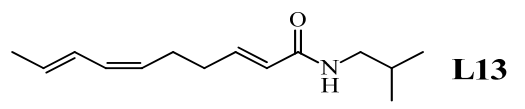

spilanthol<smiles>COc1ccc(CCC(=O)O)cc1OC</smiles>

3,4-dimethoxybenzenepropionic acid<smiles>CCC(=O)c1cc2c(cc1OC)OCO2</smiles>

kakuol methyl ether

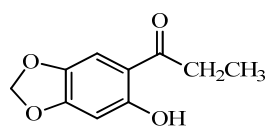

kakuol<smiles>COc1cc(CCC(=O)O)ccc1O</smiles>

hydroferulic acid
2 benzene derivatives<smiles>COc1cc(C)cc(OC)c1OC</smiles>

3,4,5-trimethoxytoluene

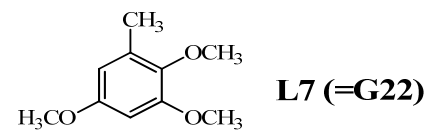

2,3,5-trimethoxytoluene

\subsubsection{Fragmentation Behaviors of Four Reference Compounds in APCI-MS ${ }^{\mathrm{n}}$}

Fragmentation behavior of l-asarinin (molecular formula: $\mathrm{C}_{20} \mathrm{H}_{18} \mathrm{O}_{6}$; exact mass: 354.1103). The quasi-molecular ion $[\mathrm{M}+\mathrm{H}]^{+}$of $l$-asarinin was observed at $m / z 355.1178$, and the base peak ion $\left[\mathrm{M}+\mathrm{H}_{-}-\mathrm{H}_{2} \mathrm{O}\right]^{+}$at $m / z 337.1045$ was observed in $\mathrm{MS}$ spectrum. In $\mathrm{MS}^{2}$ spectrum, the precursor ion $\left[\mathrm{M}+\mathrm{H}-\mathrm{H}_{2} \mathrm{O}\right]^{+}$at $m / z 337.1045\left(\mathrm{C}_{20} \mathrm{H}_{17} \mathrm{O}_{5}\right)$ fragmented into product ions at $m / z 319.0955\left(\mathrm{C}_{20} \mathrm{H}_{15} \mathrm{O}_{4}\right.$, $\left.\left[\mathrm{M}+\mathrm{H}^{-}-2 \mathrm{H}_{2} \mathrm{O}\right]^{+}\right), \quad m / z \quad 289.0846 \quad\left(\mathrm{C}_{19} \mathrm{H}_{13} \mathrm{O}_{3}, \quad\left[\mathrm{M}+\mathrm{H}-2 \mathrm{H}_{2} \mathrm{O}-\mathrm{CH}_{2} \mathrm{O}\right]^{+}\right), \quad m / z \quad 261.0901 \quad\left(\mathrm{C}_{18} \mathrm{H}_{13} \mathrm{O}_{2}\right.$, $\left.\left[\mathrm{M}+\mathrm{H}-2 \mathrm{H}_{2} \mathrm{O}-\mathrm{CH}_{2} \mathrm{O}-\mathrm{CO}\right]^{+}\right), \quad m / z \quad 231.0803 \quad\left(\mathrm{C}_{17} \mathrm{H}_{11} \mathrm{O}, \quad\left[\mathrm{M}+\mathrm{H}-2 \mathrm{H}_{2} \mathrm{O}-\mathrm{CH}_{2} \mathrm{O}-\mathrm{CO}-\mathrm{CH}_{2} \mathrm{O}\right]^{+}\right), \quad m / z$ 203.0885 $\left(\mathrm{C}_{16} \mathrm{H}_{11},\left[\mathrm{M}+\mathrm{H}-2 \mathrm{H}_{2} \mathrm{O}-\mathrm{CH}_{2} \mathrm{O}-\mathrm{CO}-\mathrm{CH}_{2} \mathrm{O}-\mathrm{CO}\right]^{+}\right)$, and $m / z 135.0434\left[\mathrm{C}_{8} \mathrm{H}_{7} \mathrm{O}_{2}\right]^{+}$. The ion at $m / z$ $135.0434\left[\mathrm{C}_{8} \mathrm{H}_{7} \mathrm{O}_{2}\right]^{+}$is a very important characteristic fragment ion for identifying the structure of the aryl group in furofuran lignans, which indicated that the aryl group was 3,4-methylenedioxyphenyl [13,14]. 
In the $\mathrm{MS}^{3}$ spectrum, the precursor ion $\left[\mathrm{M}+\mathrm{H}-2 \mathrm{H}_{2} \mathrm{O}-\mathrm{CH}_{2} \mathrm{O}\right]^{+}$at $m / z 289.0846$ further fragmented into product ions at $m / z 261.0929\left(\mathrm{C}_{18} \mathrm{H}_{13} \mathrm{O}_{2},\left[\mathrm{M}+\mathrm{H}-2 \mathrm{H}_{2} \mathrm{O}-\mathrm{CH}_{2} \mathrm{O}-\mathrm{CO}\right]^{+}\right), m / z 231.0795\left(\mathrm{C}_{17} \mathrm{H}_{11} \mathrm{O}\right.$, $\left.\left[\mathrm{M}+\mathrm{H}-2 \mathrm{H}_{2} \mathrm{O}-\mathrm{CH}_{2} \mathrm{O}-\mathrm{CO}-\mathrm{CH}_{2} \mathrm{O}\right]^{+}\right)$, and $m / z 203.0864\left(\mathrm{C}_{16} \mathrm{H}_{11},\left[\mathrm{M}+\mathrm{H}-2 \mathrm{H}_{2} \mathrm{O}-\mathrm{CH}_{2} \mathrm{O}-\mathrm{CO}-\mathrm{CH}_{2} \mathrm{O}-\mathrm{CO}\right]^{+}\right)$.

Fragmentation behavior of epipinoresinol (molecular formula: $\mathrm{C}_{20} \mathrm{H}_{22} \mathrm{O}_{6}$; exact mass: 358.1416). In MS spectrum, the quasi-molecular ion $[\mathrm{M}+\mathrm{H}]^{+}$of epipinoresinol was observed at $\mathrm{m} / z$ 359.1444, and the base peak ion $\left[\mathrm{M}+\mathrm{H}-\mathrm{H}_{2} \mathrm{O}\right]^{+}$at $m / z 341.1363\left(\mathrm{C}_{20} \mathrm{H}_{21} \mathrm{O}_{5}\right)$ was observed. In the $\mathrm{MS}^{2}$ spectrum, the precursor ion $\left[\mathrm{M}+\mathrm{H}-\mathrm{H}_{2} \mathrm{O}\right]^{+}$at $m / z 341.1363\left(\mathrm{C}_{20} \mathrm{H}_{21} \mathrm{O}_{5}\right)$ fragmented into product ions at $m / z 323.1258$ $\left(\mathrm{C}_{20} \mathrm{H}_{19} \mathrm{O}_{4}, \quad\left[\mathrm{M}+\mathrm{H}-2 \mathrm{H}_{2} \mathrm{O}\right]^{+}\right), \quad m / z \quad 291.0994 \quad\left(\mathrm{C}_{19} \mathrm{H}_{15} \mathrm{O}_{3}, \quad\left[\mathrm{M}+\mathrm{H}-2 \mathrm{H}_{2} \mathrm{O}-\mathrm{CH}_{3} \mathrm{OH}\right]^{+}\right), \quad m / z \quad 263.1042$ $\left(\mathrm{C}_{18} \mathrm{H}_{15} \mathrm{O}_{2},\left[\mathrm{M}+\mathrm{H}-2 \mathrm{H}_{2} \mathrm{O}-\mathrm{CH}_{3} \mathrm{OH}-\mathrm{CO}\right]^{+}\right), m / z 259.0734\left(\mathrm{C}_{18} \mathrm{H}_{11} \mathrm{O}_{2}, \quad\left[\mathrm{M}+\mathrm{H}-2 \mathrm{H}_{2} \mathrm{O}-2 \mathrm{CH}_{3} \mathrm{OH}\right]^{+}\right)$, and $m / z 137.0596\left(\left[\mathrm{C}_{8} \mathrm{H}_{9} \mathrm{O}_{2}\right]^{+}\right)$. The successive losses of two $\mathrm{CH}_{3} \mathrm{OH}$ from the ion $m / z 323.1258$ indicated that there were two $o$-methoxy hydroxyphenyls in the structure, and the characteristic ion at $\mathrm{m} / \mathrm{z}$ $137.0596\left(\left[\mathrm{C}_{8} \mathrm{H}_{9} \mathrm{O}_{2}\right]^{+}\right)$further confirmed this. In $\mathrm{MS}^{3}$ spectrum, the precursor ion $\left[\mathrm{M}+\mathrm{H}-2 \mathrm{H}_{2} \mathrm{O}\right]^{+}$at $m / z$ 323.1186 generated product ions at $\mathrm{m} / \mathrm{z} 291.1024\left[\mathrm{M}+\mathrm{H}-2 \mathrm{H}_{2} \mathrm{O}-\mathrm{CH}_{3} \mathrm{OH}\right]^{+}$and $\mathrm{m} / \mathrm{z} 263.1052$ $\left[\mathrm{M}+\mathrm{H}-2 \mathrm{H}_{2} \mathrm{O}-\mathrm{CH}_{3} \mathrm{OH}-\mathrm{CO}\right]^{+}$; the precursor ion at $\mathrm{m} / \mathrm{z} 291.0094\left(\mathrm{C}_{19} \mathrm{H}_{15} \mathrm{O}_{3}\right)$ gave product ions at $\mathrm{m} / \mathrm{z}$ $276.0793\left(\mathrm{C}_{18} \mathrm{H}_{12} \mathrm{O}_{3}\right)$ by losing a methyl radical, which also indicated that there was a methoxyl group in the molecule.

Fragmentation behavior of kakuol (molecular formula: $\mathrm{C}_{10} \mathrm{H}_{10} \mathrm{O}_{4}$; exact mass: 194.0579). In MS spectrum, kakuol showed quasi-molecular ion $[\mathrm{M}+\mathrm{H}]^{+}$at $m / z$ 195.0648. The quasi-molecular ion $[\mathrm{M}+\mathrm{H}]^{+}$gave fragment ions at $m / z 177.0522\left(\mathrm{C}_{10} \mathrm{H}_{9} \mathrm{O}_{3},\left[\mathrm{M}+\mathrm{H}-\mathrm{H}_{2} \mathrm{O}\right]^{+}\right), m / z \quad 166.0109\left(\mathrm{C}_{8} \mathrm{H}_{6} \mathrm{O}_{4}\right.$, $\left.\left[\mathrm{M}+\mathrm{H}^{-} \mathrm{C}_{2} \mathrm{H}_{5}\right]^{+}\right), m / z 147.0418\left(\mathrm{C}_{9} \mathrm{H}_{7} \mathrm{O}_{2},\left[\mathrm{M}+\mathrm{H}-\mathrm{H}_{2} \mathrm{O}-\mathrm{CH}_{2} \mathrm{O}\right]^{+}\right), m / z 139.0386\left(\mathrm{C}_{7} \mathrm{H}_{7} \mathrm{O}_{3},\left[\mathrm{M}+\mathrm{H}-\mathrm{C}_{3} \mathrm{H}_{4} \mathrm{O}\right]^{+}\right)$, $m / z 137.0179\left(\mathrm{C}_{7} \mathrm{H}_{5} \mathrm{O}_{3},\left[\mathrm{M}+\mathrm{H}-\mathrm{C}_{3} \mathrm{H}_{6} \mathrm{O}\right]^{+}\right), m / z 119.0478\left(\mathrm{C}_{8} \mathrm{H}_{7} \mathrm{O},\left[\mathrm{M}+\mathrm{H}-\mathrm{H}_{2} \mathrm{O}-\mathrm{CH}_{2} \mathrm{O}-\mathrm{CO}\right]^{+}\right), m / z$ $109.0261\left(\mathrm{C}_{6} \mathrm{H}_{5} \mathrm{O}_{2},\left[\mathrm{M}+\mathrm{H}-\mathrm{C}_{3} \mathrm{H}_{4} \mathrm{O}-\mathrm{CH}_{2} \mathrm{O}\right]^{+}\right.$or $\left.\left[\mathrm{M}+\mathrm{H}-\mathrm{C}_{3} \mathrm{H}_{6} \mathrm{O}-\mathrm{CO}\right]^{+}\right)$, and $m / z 95.0509\left(\mathrm{C}_{6} \mathrm{H}_{7} \mathrm{O}\right)$ in $\mathrm{MS}^{2}$ spectrum.

Fragmentation behavior of kakuol methyl ether (molecular formula: $\mathrm{C}_{11} \mathrm{H}_{12} \mathrm{O}_{4}$; exact mass: 208.0736). It showed $[\mathrm{M}+\mathrm{H}]^{+}$at $m / z 209.0801$ in $\mathrm{MS}$ spectrum, and the characteristic product ions at $m / z 191.0717\left(\mathrm{C}_{11} \mathrm{H}_{11} \mathrm{O}_{3},\left[\mathrm{M}+\mathrm{H}-\mathrm{H}_{2} \mathrm{O}\right]^{+}\right), m / z 176.0460\left(\mathrm{C}_{10} \mathrm{H}_{8} \mathrm{O}_{3},\left[\mathrm{M}+\mathrm{H}-\mathrm{H}_{2} \mathrm{O}-\mathrm{CH}_{3}\right]^{+}\right), m / z 161.0673$ $\left(\mathrm{C}_{10} \mathrm{H}_{9} \mathrm{O}_{2},\left[\mathrm{M}+\mathrm{H}-\mathrm{H}_{2} \mathrm{O}-\mathrm{CH}_{2} \mathrm{O}\right]^{+}\right), 133.0614\left(\mathrm{C}_{9} \mathrm{H}_{9} \mathrm{O},\left[\mathrm{M}+\mathrm{H}-\mathrm{H}_{2} \mathrm{O}-\mathrm{CH}_{2} \mathrm{O}-\mathrm{CO}\right]^{+}\right)$were observed in $\mathrm{MS}^{2}$ spectrum of $[\mathrm{M}+\mathrm{H}]^{+}$. The precursor ion $\left[\mathrm{M}+\mathrm{H}-\mathrm{H}_{2} \mathrm{O}-\mathrm{CH}_{3}\right]^{+}$at $\mathrm{m} / \mathrm{z} 176.0460$ gave a product ion at $\mathrm{m} / \mathrm{z}$ $147.0423\left(\mathrm{C}_{9} \mathrm{H}_{7} \mathrm{O}_{2},\left[\mathrm{M}+\mathrm{H}-\mathrm{H}_{2} \mathrm{O}-\mathrm{CH}_{3}-\mathrm{CHO}\right]^{+}\right)$in $\mathrm{MS}^{3}$ spectrum.

\subsubsection{Characterization of Eight Absorbed Lignans of AR by HPLC-APCI-IT-TOF-MS ${ }^{\mathrm{n}}$}

Most lignans in AR belong to furofuran type. The structure skeleton, common substituent groups and diagnostic fragment ions of this type lignan are summarized in Table 3 according to our research and literature data [13-16]. 
Table 3. The structure skeleton, common substituent groups and diagnostic positive APCI-MS ${ }^{\mathrm{n}}$ fragment ions of furofuran lignans.

\begin{tabular}{|c|c|c|c|c|}
\hline \multirow[t]{9}{*}{$\mathrm{R}_{1}$} & $\mathrm{R}_{2}$ & $\mathrm{R}_{1}$ diagnostic ion & $\mathrm{R}_{2}$ diagnostic ion & MS base peak ion $\left[\mathrm{M}+\mathrm{H}-\mathrm{H}_{2} \mathrm{O}\right]^{+}$ \\
\hline & & $135.04\left(\mathrm{C}_{8} \mathrm{H}_{7} \mathrm{O}_{2}\right)$ & $135.04\left(\mathrm{C}_{8} \mathrm{H}_{7} \mathrm{O}_{2}\right)$ & $337.10\left(\mathrm{C}_{20} \mathrm{H}_{17} \mathrm{O}_{5}\right)$ \\
\hline & & $135.04\left(\mathrm{C}_{8} \mathrm{H}_{7} \mathrm{O}_{2}\right)$ & $137.06\left(\mathrm{C}_{8} \mathrm{H}_{9} \mathrm{O}_{2}\right)$ & $339.12\left(\mathrm{C}_{20} \mathrm{H}_{19} \mathrm{O}_{5}\right)$ \\
\hline & & $137.06\left(\mathrm{C}_{8} \mathrm{H}_{9} \mathrm{O}_{2}\right)$ & $137.06\left(\mathrm{C}_{8} \mathrm{H}_{9} \mathrm{O}_{2}\right)$ & $341.14\left(\mathrm{C}_{20} \mathrm{H}_{21} \mathrm{O}_{5}\right)$ \\
\hline & & $123.04\left(\mathrm{C}_{7} \mathrm{H}_{7} \mathrm{O}_{2}\right)$ & $123.04\left(\mathrm{C}_{7} \mathrm{H}_{7} \mathrm{O}_{2}\right)$ & $313.10\left(\mathrm{C}_{18} \mathrm{H}_{17} \mathrm{O}_{5}\right)$ \\
\hline & & $151.07\left(\mathrm{C}_{9} \mathrm{H}_{11} \mathrm{O}_{2}\right)$ & $151.07\left(\mathrm{C}_{9} \mathrm{H}_{11} \mathrm{O}_{2}\right)$ & $369.17\left(\mathrm{C}_{22} \mathrm{H}_{25} \mathrm{O}_{5}\right)$ \\
\hline & & $137.06\left(\mathrm{C}_{8} \mathrm{H}_{9} \mathrm{O}_{2}\right)$ & $167.07\left(\mathrm{C}_{9} \mathrm{H}_{11} \mathrm{O}_{3}\right)$ & $371.15\left(\mathrm{C}_{21} \mathrm{H}_{23} \mathrm{O}_{6}\right)$ \\
\hline & & $135.04\left(\mathrm{C}_{8} \mathrm{H}_{7} \mathrm{O}_{2}\right)$ & $167.07\left(\mathrm{C}_{9} \mathrm{H}_{11} \mathrm{O}_{3}\right)$ & $369.13\left(\mathrm{C}_{21} \mathrm{H}_{21} \mathrm{O}_{6}\right)$ \\
\hline & & $135.04\left(\mathrm{C}_{8} \mathrm{H}_{7} \mathrm{O}_{2}\right)$ & $153.05\left(\mathrm{C}_{8} \mathrm{H}_{9} \mathrm{O}_{3}\right)$ & $355.12\left(\mathrm{C}_{20} \mathrm{H}_{19} \mathrm{O}_{6}\right)$ \\
\hline
\end{tabular}

Identification of $\boldsymbol{L 1}, \boldsymbol{L 2}, \boldsymbol{L 5}$. All of them showed $[\mathrm{M}+\mathrm{H}]^{+}$at $m / z$ 359.14, and the base peak ion in MS was at $m / z$ 341.14, indicating that their molecular formulae were $\mathrm{C}_{20} \mathrm{H}_{22} \mathrm{O}_{6}$. In $\mathrm{MS}^{2}$ spectra of them, the diagnostic ions at $m / z 291.10\left(\mathrm{C}_{19} \mathrm{H}_{15} \mathrm{O}_{3},\left[\mathrm{M}+\mathrm{H}-2 \mathrm{H}_{2} \mathrm{O}-\mathrm{CH}_{3} \mathrm{OH}\right]^{+}\right), m / z 259.07\left(\mathrm{C}_{18} \mathrm{H}_{11} \mathrm{O}_{2}\right.$, $\left.\left[\mathrm{M}+\mathrm{H}-2 \mathrm{H}_{2} \mathrm{O}-2 \mathrm{CH}_{3} \mathrm{OH}\right]^{+}\right)$, and $m / z 137.06\left(\mathrm{C}_{8} \mathrm{H}_{9} \mathrm{O}_{2}\right)$ indicated that two aryl groups in $\mathbf{L 1}, \mathbf{L} 2$ and $\mathbf{L 5}$ were $o$-methoxy hydroxyphenyl. Further, $\mathbf{L 5}$ was confirmed to be epipinoresinol by comparison with the reference compound, and $\mathbf{L} \mathbf{1}$ and $\mathbf{L} \mathbf{2}$ were tentatively identified as epipinoresinol isomers.

Identification of $\mathbf{L 3}$ and L10. The base peak ions of them were $m / z 339.12$ in MS, and the diagnostic ions of two aryl groups were observed at $m / z 135.04\left(\mathrm{C}_{8} \mathrm{H}_{7} \mathrm{O}_{2}\right)$ and $m / z 137.06\left(\mathrm{C}_{8} \mathrm{H}_{9} \mathrm{O}_{2}\right)$, accompanied with the ion at $m / z 289.09\left[\mathrm{M}+\mathrm{H}-2 \mathrm{H}_{2} \mathrm{O}-\mathrm{CH}_{3} \mathrm{OH}\right]^{+}$in $\mathrm{MS}^{2}$. This indicated that one aryl was $o$-methoxy hydroxyphenyl and the other was 3,4-methylenedioxyphenyl. Accordingly, L10 was 
identified as xanthoxylol by comparison with the reference compound, and L3 was tentatively identified as a xanthoxylol isomer.

Identification of $\boldsymbol{L 8}$. It showed $[\mathrm{M}+\mathrm{H}]^{+}$at $\mathrm{m} / \mathrm{z}$ 387.14, and the base peak ion in MS was at $\mathrm{m} / \mathrm{z}$ 369.13 , indicating that its molecular formula was $\mathrm{C}_{21} \mathrm{H}_{22} \mathrm{O}_{7}$. The diagnostic ions of two aryl groups were observed at $m / z 135.04\left(\mathrm{C}_{8} \mathrm{H}_{7} \mathrm{O}_{2}\right)$ and $m / z 167.07\left(\mathrm{C}_{9} \mathrm{H}_{11} \mathrm{O}_{3}\right)$, accompanied with the ion at $\mathrm{m} / \mathrm{z}$ $319.10\left[\mathrm{M}+\mathrm{H}-2 \mathrm{H}_{2} \mathrm{O}-\mathrm{CH}_{3} \mathrm{OH}\right]^{+}$in $\mathrm{MS}^{2}$. This indicated that one aryl was $o$-methoxy dihydroxyphenyl and the other was 3,4-methylenedioxyphenyl. Therefore, $\mathbf{L 8}$ was tentatively identified as $(1 R, 2 S, 5 R, 6 R)-5^{\prime}-O$-methylpluviatilol [17], which was isolated from Asarum sieboldii.

Identification of $\mathbf{L} 14$ and $\mathbf{L 1 5}$. L15 was confirmed to be $l$-asarinin by comparison with reference compound. $\mathbf{L 1 4}$ was an isomer of $\mathbf{L 1 5}$, which showed $[\mathrm{M}+\mathrm{H}]^{+}$at $\mathrm{m} / z 355.12$ and the base peak ion $\left[\mathrm{M}+\mathrm{H}-\mathrm{H}_{2} \mathrm{O}\right]^{+}$at $\mathrm{m} / \mathrm{z} 337.10$ in MS. The $\mathrm{MS}^{2}$ of $\mathbf{L 1 5}$ showed characteristic product ions at $\mathrm{m} / \mathrm{z} 289.08$ $\left(\mathrm{C}_{19} \mathrm{H}_{13} \mathrm{O}_{3},\left[\mathrm{M}+\mathrm{H}-2 \mathrm{H}_{2} \mathrm{O}-\mathrm{CH}_{2} \mathrm{O}\right]^{+}\right)$and $m / z 135.0434\left[\mathrm{C}_{8} \mathrm{H}_{7} \mathrm{O}_{2}\right]^{+}$, which was consistent with that of $l$-sesamin [15], so $\mathbf{L 1 5}$ was tentatively identified as $l$-sesamin.

\subsubsection{Characterization of Nine Absorbed N-alkylamides of AR by HPLC-APCI-IT-TOF-MS ${ }^{\mathrm{n}}$}

The nine absorbed constituents (L13, L16-L23) were identified as $N$-isobutylamides, because of their characteristic neutral losses of $56 \mathrm{Da}\left(\mathrm{C}_{4} \mathrm{H}_{8}\right), 73 \mathrm{Da}\left(\mathrm{C}_{4} \mathrm{H}_{11} \mathrm{~N}\right), 99 \mathrm{Da}\left(\mathrm{C}_{5} \mathrm{H}_{9} \mathrm{NO}\right)$ and $101 \mathrm{Da}$ $\left(\mathrm{C}_{5} \mathrm{H}_{11} \mathrm{NO}\right)$ from $N$-isobutylamide were observed in their $\mathrm{MS}^{\mathrm{n}}$ spectra [18-21].

Identification of L16-L19. All of them showed $[\mathrm{M}+\mathrm{H}]^{+}$at $\mathrm{m} / z$ 248.20, which indicated that their molecular formulae were $\mathrm{C}_{16} \mathrm{H}_{25} \mathrm{NO}$. The fragment ions at $m / z 192.14\left(\mathrm{C}_{12} \mathrm{H}_{18} \mathrm{NO},\left[\mathrm{M}+\mathrm{H}-\mathrm{C}_{4} \mathrm{H}_{8}\right]^{+}\right), m / z$ $175.11\left(\mathrm{C}_{12} \mathrm{H}_{15} \mathrm{O},\left[\mathrm{M}+\mathrm{H}-\mathrm{C}_{4} \mathrm{H}_{11} \mathrm{~N}\right]^{+}\right), m / z 167.13\left(\mathrm{C}_{10} \mathrm{H}_{17} \mathrm{NO},\left[\mathrm{M}+\mathrm{H}-\mathrm{C}_{6} \mathrm{H}_{9}\right]^{+}\right), m / z 166.12\left(\mathrm{C}_{10} \mathrm{H}_{16} \mathrm{NO}\right.$, $\left.\left[\mathrm{M}+\mathrm{H}^{-} \mathrm{C}_{6} \mathrm{H}_{10}\right]^{+}\right), m / z 149.13\left(\mathrm{C}_{11} \mathrm{H}_{17},\left[\mathrm{M}+\mathrm{H}-\mathrm{C}_{5} \mathrm{H}_{9} \mathrm{NO}\right]^{+}\right), m / z 147.12\left(\mathrm{C}_{11} \mathrm{H}_{15},\left[\mathrm{M}+\mathrm{H}-\mathrm{C}_{5} \mathrm{H}_{11} \mathrm{NO}\right]^{+}\right)$, $m / z 133.07\left(\mathrm{C}_{9} \mathrm{H}_{9} \mathrm{O},\left[\mathrm{M}+\mathrm{H}-\mathrm{C}_{4} \mathrm{H}_{11} \mathrm{~N}-\mathrm{C}_{3} \mathrm{H}_{6}\right]^{+}\right), m / z 121.10\left(\mathrm{C}_{9} \mathrm{H}_{13},\left[\mathrm{M}+\mathrm{H}-\mathrm{C}_{7} \mathrm{H}_{13} \mathrm{NO}\right]^{+}\right)$, and $m / z 107.08$ $\left(\mathrm{C}_{8} \mathrm{H}_{11},\left[\mathrm{M}+\mathrm{H}-\mathrm{C}_{7} \mathrm{H}_{13} \mathrm{NO}\right]^{+}\right)$were observed in their $\mathrm{MS}^{2}$ spectra, which indicated that they had the degree of unsaturation of 5 and an alkyl chain of 12 carbons, and they were $N$-isobutyl-2,4,8,10dodecatetraenamides [19]. Their UV spectra exhibited $\lambda_{\max }$ at $259 \mathrm{~nm}$, which further confirmed that they were 2,4-diene alkamides. Besides, the peak areas of L16 and L17 were bigger than those of $\mathbf{L 1 8}$ and L19, indicating that L16 and L17 were more abundant than L18 and L19. Because $N$-isobutyl$2 E, 4 E, 8 Z, 10 E$-dodecatetraenamide and $N$-isobutyl-2E,4E,8Z,10Z-dodecatetraenamide were isolated from Asari Radix et Rhizoma [22], and the $2 E, 4 E, 8 Z, 10 Z$-isomer elute before $2 E, 4 E, 8 Z, 10 E$-isomer in HPLC [20], L16 was tentatively identified as $N$-isobutyl-2E,4E,8Z,10Z-dodecatetraenamide and $\mathbf{L 1 7}$ as $N$-isobutyl-2E,4E,8Z,10E-dodecatetraenamide. Besides, $\mathbf{L 1 8}$ and $\mathbf{L 1 9}$ were tentatively identified as $N$-isobutyl-2,4,8,10-dodecatetraenamide isomers.

Identification of $\mathbf{L 2 0}$. It showed $[\mathrm{M}+\mathrm{H}]^{+}$at $\mathrm{m} / \mathrm{z} 250.2150$, indicating the molecular formula of $\mathrm{C}_{16} \mathrm{H}_{27} \mathrm{NO}$. Its UV spectrum exhibited $\lambda_{\max }$ at $262 \mathrm{~nm}$, which indicated that $\mathbf{L 2 0}$ was a 2,4-diene alkamide. The fragment ions at $m / z 194.1625\left(\mathrm{C}_{12} \mathrm{H}_{20} \mathrm{NO},\left[\mathrm{M}+\mathrm{H}-\mathrm{C}_{4} \mathrm{H}_{8}\right]^{+}\right), m / z 177.1164\left(\mathrm{C}_{12} \mathrm{H}_{17} \mathrm{O}\right.$, $\left.\left[\mathrm{M}+\mathrm{H}-\mathrm{C}_{4} \mathrm{H}_{11} \mathrm{~N}\right]^{\dagger}\right), m / z 167.1302\left(\mathrm{C}_{10} \mathrm{H}_{17} \mathrm{NO},\left[\mathrm{M}+\mathrm{H}-\mathrm{C}_{6} \mathrm{H}_{11}\right]^{+}\right), m / z \quad 152.1070\left(\mathrm{C}_{9} \mathrm{H}_{14} \mathrm{NO},\left[\mathrm{M}+\mathrm{H}-\mathrm{C}_{7} \mathrm{H}_{14}\right]^{+}\right)$, and $m / z$ 149.1398 $\left(\mathrm{C}_{11} \mathrm{H}_{17},\left[\mathrm{M}+\mathrm{H}-\mathrm{C}_{5} \mathrm{H}_{11} \mathrm{NO}\right]^{+}\right)$in $\mathrm{MS}^{2}$ of $\mathbf{~} 20$ indicated that it had the degree of 
unsaturation of 4 and an alkyl chain of 12 carbons, and it was $N$-isobutyl-dodecatrienamide. By comparison with the literature [20], L20 was tentatively identified as $N$-isobutyl-2,4,8-dodecatrienamide.

Identification of $\mathbf{L 2 1}$. It showed $[\mathrm{M}+\mathrm{H}]^{+}$at $\mathrm{m} / \mathrm{z} 274.2164$, indicating the molecular formula of $\mathrm{C}_{18} \mathrm{H}_{27} \mathrm{NO}$. The UV spectrum of $\mathbf{L 2 1}$ exhibited $\lambda_{\max }$ at $263 \mathrm{~nm}$, which implied that it was a 2,4-diene alkamide. The characteristic fragment ions at $m / z 201.1332\left(\mathrm{C}_{14} \mathrm{H}_{17} \mathrm{O},\left[\mathrm{M}+\mathrm{H}-\mathrm{C}_{4} \mathrm{H}_{11} \mathrm{~N}\right]^{+}\right), m / z 175.1411$ $\left(\mathrm{C}_{13} \mathrm{H}_{19},\left[\mathrm{M}+\mathrm{H}-\mathrm{C}_{5} \mathrm{H}_{9} \mathrm{NO}\right]^{+}\right)$, and $m / z 173.1332\left(\mathrm{C}_{13} \mathrm{H}_{17},\left[\mathrm{M}+\mathrm{H}-\mathrm{C}_{5} \mathrm{H}_{11} \mathrm{NO}\right]^{+}\right)$in $\mathrm{MS}^{2}$ spectra of $\mathbf{L 2 1}$ indicated that it had the degree of unsaturation of 6 and an alkyl chain of 14 carbons, and it was $N$-isobutyl-tetradecapentaenamide. Therefore, $\mathbf{L 2 1}$ was tentatively identified as $N$-isobutyl-2,4,8,10,12tetradecapentaenamide.

Identification of L22. It showed $[\mathrm{M}+\mathrm{H}]^{+}$at $\mathrm{m} / \mathrm{z}$ 276.2322, which indicated that its molecular formula was $\mathrm{C}_{18} \mathrm{H}_{29} \mathrm{NO}$. The UV spectrum of $\mathbf{L 2 2}$ exhibited $\lambda_{\max }$ at $262 \mathrm{~nm}$, which indicated that it was a 2,4-diene alkamide. The characteristic fragment ions at $m / z 220.1623\left(\mathrm{C}_{14} \mathrm{H}_{22} \mathrm{NO},\left[\mathrm{M}+\mathrm{H}-\mathrm{C}_{4} \mathrm{H}_{8}\right]^{+}\right), m / z$ $203.1457\left(\mathrm{C}_{14} \mathrm{H}_{19} \mathrm{O},\left[\mathrm{M}+\mathrm{H}-\mathrm{C}_{4} \mathrm{H}_{11} \mathrm{~N}\right]^{+}\right), \quad m / z \quad 177.1611\left(\mathrm{C}_{13} \mathrm{H}_{21},\left[\mathrm{M}+\mathrm{H}-\mathrm{C}_{5} \mathrm{H}_{9} \mathrm{NO}\right]^{+}\right), m / z \quad 175.1431$ $\left(\mathrm{C}_{13} \mathrm{H}_{19},\left[\mathrm{M}+\mathrm{H}-\mathrm{C}_{5} \mathrm{H}_{11} \mathrm{NO}\right]^{+}\right), m / z 167.1297\left(\mathrm{C}_{10} \mathrm{H}_{17} \mathrm{NO},\left[\mathrm{M}+\mathrm{H}-\mathrm{C}_{8} \mathrm{H}_{13}\right]^{+}\right), m / z$ 135.1181 $\left(\mathrm{C}_{10} \mathrm{H}_{15}\right)$, and $\mathrm{m} / z 133.0970\left(\mathrm{C}_{10} \mathrm{H}_{13}\right)$ in $\mathrm{MS}^{2}$ spectra of $\mathbf{L 2 2}$ indicated that it had the degree of unsaturation of 5 and an alkyl chain of 14 carbons, and it was $N$-isobutyl-tetradecatetraenamide. Accordingly, L22 was tentatively identified as $N$-isobutyl-2,4,8,10-tetradecapentaenamide.

Identification of L23. It showed $[\mathrm{M}+\mathrm{H}]^{+}$at $\mathrm{m} / \mathrm{z}$ 252.2317, which indicated that its molecular formula was $\mathrm{C}_{16} \mathrm{H}_{29} \mathrm{NO}$. The UV spectrum of $\mathbf{L 2 3}$ exhibited $\lambda_{\max }$ at $262 \mathrm{~nm}$, which indicated that it was a 2,4-diene alkamide. The characteristic fragment ions at $m / z 196.1744\left(\mathrm{C}_{12} \mathrm{H}_{22} \mathrm{NO},\left[\mathrm{M}+\mathrm{H}-\mathrm{C}_{4} \mathrm{H}_{8}\right]^{+}\right)$, $m / z 179.1256\left(\mathrm{C}_{12} \mathrm{H}_{19} \mathrm{O},\left[\mathrm{M}+\mathrm{H}-\mathrm{C}_{4} \mathrm{H}_{11} \mathrm{~N}\right]^{+}\right), m / z 154.1227\left(\mathrm{C}_{9} \mathrm{H}_{16} \mathrm{NO},\left[\mathrm{M}+\mathrm{H}-\mathrm{C}_{7} \mathrm{H}_{14}\right]^{+}\right)$, and $m / z 95.0480$ $\left(\mathrm{C}_{6} \mathrm{H}_{7} \mathrm{O}\right)$ in $\mathrm{MS}^{2}$ spectra of $\mathbf{L 2 3}$ indicated that it had the degree of unsaturation of 3 and an alkyl chain of 12 carbons, and it was $N$-isobutyl-2,4-dodecaenamide. Accordingly, $\mathbf{L 2 3}$ was tentatively identified as $N$-isobutyl-2,4-dodecaenamide.

Identification of L13. It showed $[\mathrm{M}+\mathrm{H}]^{+}$at $\mathrm{m} / \mathrm{z}$ 222.1842, which indicated that its molecular formula was $\mathrm{C}_{14} \mathrm{H}_{23} \mathrm{NO}$. The characteristic fragment ions at $m / z 167.1237\left(\mathrm{C}_{10} \mathrm{H}_{17} \mathrm{NO},\left[\mathrm{M}+\mathrm{H}-\mathrm{C}_{4} \mathrm{H}_{7}\right]^{+}\right)$, $m / z 166.1329\left(\mathrm{C}_{10} \mathrm{H}_{16} \mathrm{NO},\left[\mathrm{M}+\mathrm{H}-\mathrm{C}_{4} \mathrm{H}_{8}\right]^{+}\right), m / z 152.0970\left(\mathrm{C}_{9} \mathrm{H}_{14} \mathrm{NO},\left[\mathrm{M}+\mathrm{H}-\mathrm{C}_{5} \mathrm{H}_{10}\right]^{+}\right), m / z 149.0985$ $\left(\mathrm{C}_{10} \mathrm{H}_{13} \mathrm{O}\right.$, $\left.\left[\mathrm{M}+\mathrm{H}-\mathrm{C}_{4} \mathrm{H}_{11} \mathrm{~N}\right]^{+}\right)$, and $m / z$ 81.0807 $\left(\mathrm{C}_{6} \mathrm{H}_{9},\left[\mathrm{M}+\mathrm{H}-\mathrm{C}_{8} \mathrm{H}_{15} \mathrm{NO}\right]^{+}\right)$in $\mathrm{MS}^{2}$ spectra of $\mathbf{L 1 3}$ indicated that it had the degree of unsaturation of 4 and an alkyl chain of 10 carbons. According to literature [18], L13 was tentatively identified as spilanthol, i.e., $N$-isobutyl-2E,6Z,8E-decatrienamide, which was isolated from the roots and rhizomes of Asarum longerhizomatosum [23].

\subsubsection{Characterization of FourAbsorbed Phenylpropanoids of AR by HPLC-APCI-IT-TOF-MS ${ }^{\mathrm{n}}$}

Identification of $\mathbf{L 9}$ and $\mathbf{L 1 1}$. L9 and $\mathbf{L 1 1}$ were confirmed to be kakuol methyl ether and kakuol by comparing their retention times and $\mathrm{MS}^{\mathrm{n}}$ data with those of the reference compounds.

Identification of $\boldsymbol{L} 4$. It showed $[\mathrm{M}+\mathrm{H}]^{+}$at $\mathrm{m} / \mathrm{z} 211.0955$, indicating the molecular formula of $\mathrm{C}_{11} \mathrm{H}_{14} \mathrm{O}_{4}$. The precursor ion $m / z 211.0955\left(\mathrm{C}_{11} \mathrm{H}_{15} \mathrm{O}_{4},[\mathrm{M}+\mathrm{H}]^{+}\right)$gave fragment ions at $\mathrm{m} / z 193.0846$ $\left(\mathrm{C}_{11} \mathrm{H}_{13} \mathrm{O}_{3},\left[\mathrm{M}+\mathrm{H}-\mathrm{H}_{2} \mathrm{O}\right]^{+}\right), m / z 178.0611\left(\mathrm{C}_{10} \mathrm{H}_{10} \mathrm{O}_{3},\left[\mathrm{M}+\mathrm{H}-\mathrm{H}_{2} \mathrm{O}-\mathrm{CH}_{3}\right]^{+}\right), m / z \quad 161.0598\left(\mathrm{C}_{10} \mathrm{H}_{9} \mathrm{O}_{2}\right.$, 
$\left.\left[\mathrm{M}+\mathrm{H}-\mathrm{H}_{2} \mathrm{O}-\mathrm{CH}_{3} \mathrm{OH}\right]^{+}\right), m / z 137.0541\left(\mathrm{C}_{8} \mathrm{H}_{9} \mathrm{O}_{2},\left[\mathrm{M}+\mathrm{H}-\mathrm{H}_{2} \mathrm{O}-\mathrm{C}_{3} \mathrm{H}_{4} \mathrm{O}\right]^{+}\right)$and $m / z 133.0630\left(\mathrm{C}_{9} \mathrm{H}_{9} \mathrm{O}\right.$, $\left.\left[\mathrm{M}+\mathrm{H}-\mathrm{H}_{2} \mathrm{O}-\mathrm{CH}_{3} \mathrm{OH}-\mathrm{CO}\right]^{+}\right)$. According to these characteristic ions, $\mathbf{L} 4$ was tentatively identified as 3,4-dimethoxybenzenepropionic acid. The fragmentation pathways of $\mathbf{L} \mathbf{4}$ are proposed in Figure 7.

Figure 7. Proposed fragmentation pathways of 3,4-dimethoxybenzenepropionic acid (L4) in positive ion APCI-MS.

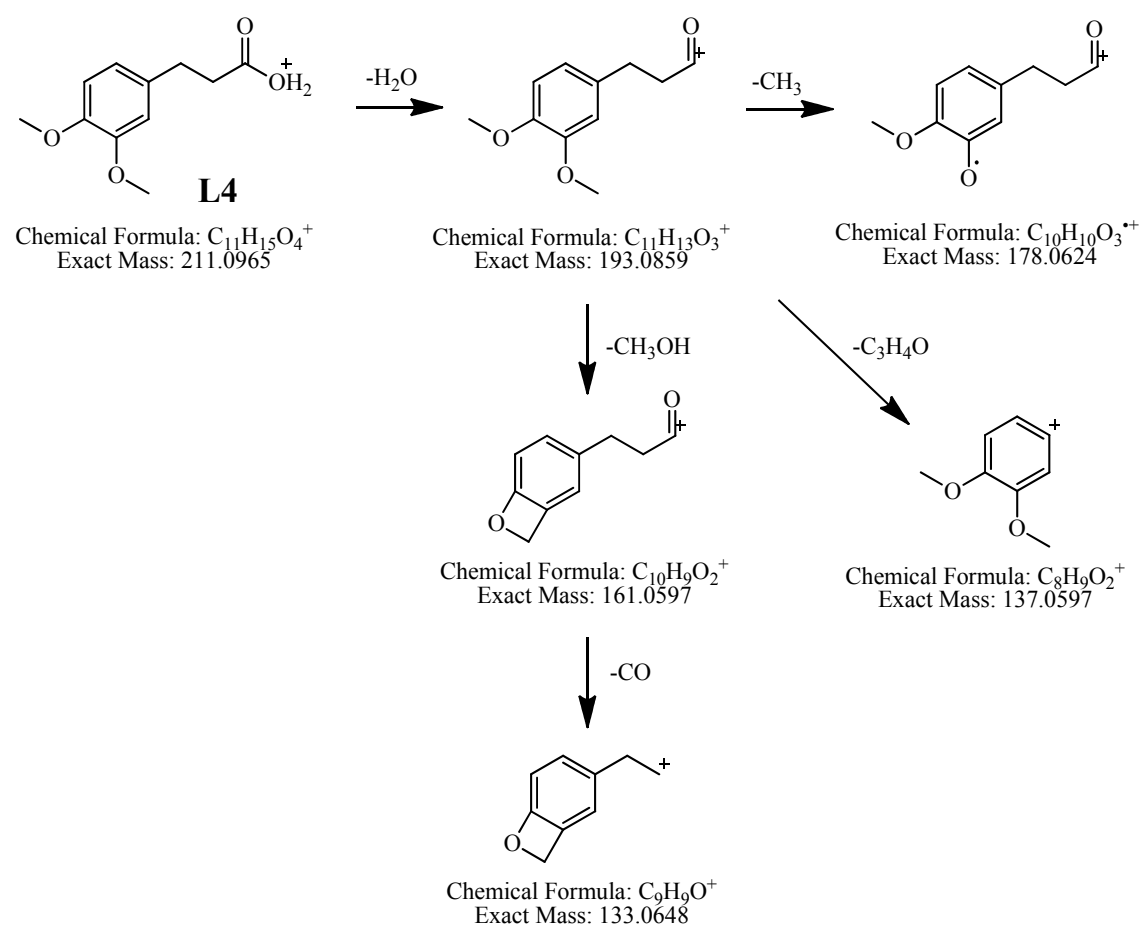

Identification of L12. It showed $[\mathrm{M}+\mathrm{H}]^{+}$at $\mathrm{m} / \mathrm{z}$ 197.0804, indicating the molecular formula of $\mathrm{C}_{10} \mathrm{H}_{12} \mathrm{O}_{4}$. The precursor ion $\mathrm{m} / z 197.0804\left(\mathrm{C}_{10} \mathrm{H}_{13} \mathrm{O}_{4},[\mathrm{M}+\mathrm{H}]^{+}\right)$gave fragment ions at $\mathrm{m} / z 179.0720$ $\left(\mathrm{C}_{10} \mathrm{H}_{11} \mathrm{O}_{3}, \quad\left[\mathrm{M}+\mathrm{H}-\mathrm{H}_{2} \mathrm{O}\right]^{+}\right), \quad m / z \quad 155.0732\left(\mathrm{C}_{8} \mathrm{H}_{11} \mathrm{O}_{3}, \quad\left[\mathrm{M}+\mathrm{H}-\mathrm{C}_{2} \mathrm{H}_{2} \mathrm{O}\right]^{+}\right), \quad m / z \quad 151.0756\left(\mathrm{C}_{9} \mathrm{H}_{11} \mathrm{O}_{2}\right.$, $\left.\left[\mathrm{M}+\mathrm{H}-\mathrm{H}_{2} \mathrm{O}-\mathrm{CO}\right]^{+}\right), \quad m / z \quad 137.0575 \quad\left(\mathrm{C}_{8} \mathrm{H}_{9} \mathrm{O}_{2}, \quad\left[\mathrm{M}+\mathrm{H}-\mathrm{H}_{2} \mathrm{O}-\mathrm{C}_{2} \mathrm{H}_{2} \mathrm{O}\right]^{+}\right), \quad m / z \quad 123.0894 \quad\left(\mathrm{C}_{8} \mathrm{H}_{11} \mathrm{O}\right.$, $\left.\left[\mathrm{M}+\mathrm{H}-\mathrm{H}_{2} \mathrm{O}-\mathrm{CO}-\mathrm{CO}\right]^{+}\right)$, and $m / z 91.0513\left(\mathrm{C}_{7} \mathrm{H}_{7},\left[\mathrm{M}+\mathrm{H}-\mathrm{H}_{2} \mathrm{O}-\mathrm{CO}-\mathrm{CO}-\mathrm{CH}_{3} \mathrm{OH}\right]^{+}\right)$. According to these characteristic ions, $\mathbf{L 1 2}$ was tentatively identified as hydroferulic acid. The fragmentation pathways of $\mathbf{L 1 2}$ are proposed in Figure 8.

Figure 8. Proposed fragmentation pathways of hydroferulic acid (L12) in positive ion APCI-MS.

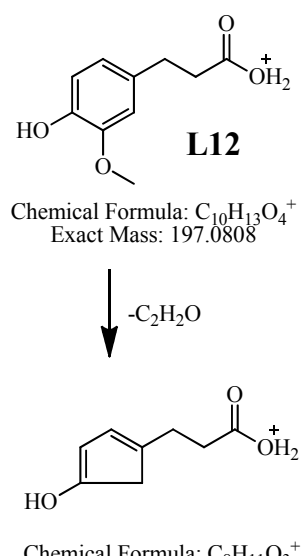

Exact Foss: 155.0703<smiles>COc1cc(CCC=O)ccc1OCCO</smiles>

Chemical Formula: $\mathrm{C}_{10} \mathrm{H}_{11} \mathrm{O}_{3}$ Exact Mass: 179.0703

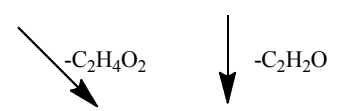<smiles>COc1cc(I)ccc1O</smiles>

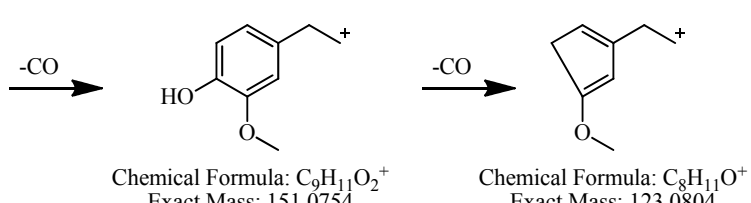

Exact Mass: 151.0754 Exact Mass: 123.0804

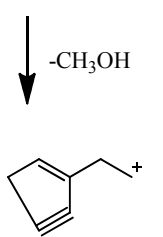




\subsubsection{Characterization of Two Absorbed Benzene Derivatives of AR by HPLC-APCI-IT-TOF-MS ${ }^{\mathrm{n}}$}

Identification of $\mathbf{L 6}$ and $\mathbf{L}$ 7. Both of them showed $[\mathrm{M}+\mathrm{H}]^{+}$at $\mathrm{m} / \mathrm{z} 183.10$, indicating that their molecular formulae were $\mathrm{C}_{10} \mathrm{H}_{14} \mathrm{O}_{3}$. The fragment ions at $m / z 168.07\left(\mathrm{C}_{9} \mathrm{H}_{12} \mathrm{O}_{3},\left[\mathrm{M}+\mathrm{H}-\mathrm{CH}_{3}\right]^{+}\right), m / z$ $152.08\left(\mathrm{C}_{9} \mathrm{H}_{12} \mathrm{O}_{2},\left[\mathrm{M}+\mathrm{H}-\mathrm{CH}_{3} \mathrm{O}\right]^{+}\right), m / z 151.07\left(\mathrm{C}_{9} \mathrm{H}_{11} \mathrm{O}_{2},\left[\mathrm{M}+\mathrm{H}-\mathrm{CH}_{3} \mathrm{OH}\right]^{+}\right)$were observed in their $\mathrm{MS}^{2}$ spectra. By comparison with reference compounds, L6 and $\mathbf{L} 7$ were unequivocally identified as 3,4,5-trimethoxytoluene and 2,3,5-trimethoxytoluene, respectively. These two compounds were also identified by HS-SPME-GC-MS technique in this study. L6 was identical compound with G20, and L7 was the same compound as $\mathbf{G 2 2}$.

\subsection{Bioactivities of the Absorbed Constituents Related to the Pharmacological Effects of AR}

Through HS-SPME-GC-MS and HPLC-APCI-IT-TOF-MS ${ }^{\mathrm{n}}$, totally 47 absorbed constituents containing 14 monoterpenes, 10 phenylpropanoids, four benzene derivatives, two alkanes, nine $\mathrm{N}$-alkylamides and eight lignans were identified in the rabbit plasma and CSF after intranasal administration of AR EtOAc extract. The numbers of identified absorbed constituents in different samples and by different methods are summarized in Table 4. It was found that these absorbed constituents were all the original constituents of AR and no metabolite was detected in the plasma and CSF. This may be because compounds avoid the gastrointestinal tract and first-pass metabolism through intranasal administration [24], indicating that these original constituents of AR might be the effective substances of AR when it is used in nasal therapy. Moreover, the molecular weight of these absorbed constituents were all less than $400 \mathrm{Da}$, and the number of the absorbed constituents having the molecular weight of less than 300 Da accounted for $83 \%$ of all the 47 absorbed constituents, which was consistent with the opinion that drugs with molecular weight lower than $300 \mathrm{Da}$ are easy to be absorbed through intranasal administration [2]. AR powder comprised of lots of constituents with various polarities, and AR EtOAc extract was prepared from AR powder by extraction with EtOAc, a nonpolar solvent. Hence, AR EtOAc extract should be the less polar portion of AR powder. However, the absorbed constituents of AR powder are all found in the absorbed constituents of AR EtOAc extract. These findings suggested that the original constituents with small molecular weights and relatively low polarities might be the effective substances of AR when it is used in nasal therapy.

Table 4. The numbers of identified absorbed constituents in plasma and CSF of AR EtOAc extract group rabbits by HS-SPME-GC-MS and HPLC-APCI-IT-TOF-MS ${ }^{\mathrm{n}}$.

\begin{tabular}{ccccc}
\hline & GC-MS & LC-MS & $\begin{array}{c}\text { Same Constituents Identified by } \\
\text { GC-MS and LC-MS }\end{array}$ & Total No. \\
\hline Plasma & 25 & 23 & $2(\mathbf{L 6}=\mathbf{G 2 0}, \mathbf{L 7}=\mathbf{G 2 2})$ & 46 \\
CSF & 25 & 5 & $2(\mathbf{L 6}=\mathbf{G 2 0}, \mathbf{L} 7=\mathbf{G} 22)$ & 28 \\
Same constituents in & $24(\mathbf{G 1}-\mathbf{G 1 3}$, & $5(\mathbf{L 6}, \mathbf{L 7}$, & $2(\mathbf{L 6}=\mathbf{G 2 0}, \mathbf{L 7}=\mathbf{G 2 2})$ & 27 \\
plasma and CSF & $\mathbf{G 1 5}, \mathbf{G 1 7}-\mathbf{G 2 6})$ & $\mathbf{L 1 5}-\mathbf{L 1 7})$ & 2 & All: $\mathbf{4 7}$ \\
Total No. & 26 & 23 & & 2 \\
\hline
\end{tabular}

To find whether the identified absorbed constituents might be the effective substances of AR, their bioactivities related to the pharmacological effects of AR were summarized based on the overall literature 
retrieval. As shown in Table 5, 33 of 47 absorbed constituents were reported to exhibit diverse bioactivities associated with the pharmacological actions of AR (detail information are listed in Supplemental Materials Table S1). We can see that one absorbed constituent can have several bioactivities (Table S1; Table 5). Namely, methyleugenol has eight bioactivities, limonene and eucalyptol have seven bioactivities; three constituents (camphor, L-borneol and $\alpha$-terpineol) have five bioactivities, six constituents have four bioactivities, three constituents have three bioactivities and six constituents have two bioactivities. These indicated that even one absorbed constituent could act on several targets simultaneously.

Table 5. Bioactivities of the absorbed constituents related to the pharmacological effects of Asari Radix et Rhizoma (AR).

\begin{tabular}{|c|c|c|}
\hline Activity (number) & Structure Type (Number) & Constituents \\
\hline \multirow{3}{*}{ Analgesic (14) } & Monoterpene (9) & $\begin{array}{l}\alpha \text {-Pinene, } \beta \text {-Pinene, Myrcene, } \alpha \text {-Phellandrene, Limonene, Eucalyptol, } \\
\text { Camphor, } l \text {-Borneol, } \alpha \text {-Terpineol }\end{array}$ \\
\hline & Phenylpropanoid (3) & Estragole, methyleugenol, 3,4-Dimethoxybenzenepropionic acid \\
\hline & $N$-alkylamide (2) & Spilanthol, $N$-Isobutyl-2E,4E, $8 Z, 10 E$-dodecatetraenamide \\
\hline \multirow{5}{*}{$\begin{array}{l}\text { Anti-inflammatory } \\
\qquad \text { (27) }\end{array}$} & Monoterpene (12) & $\begin{array}{l}\alpha \text {-Pinene, Sabinene, } \beta \text {-Pinene, Myrcene, 3-Carene, Limonene, Eucalyptol, } \\
\text { Camphor, Eucarvone, } l \text {-Borneol, Terpinen-4-ol, } \alpha \text {-Terpineol }\end{array}$ \\
\hline & Phenylpropanoid (5) & Estragole, Methyleugenol, Elemicin, Kakuol, Hydroferulic Acid \\
\hline & Benzene derivative (1) & 3,4,5-Trimethoxytoluene \\
\hline & Lignan (4) & $\begin{array}{l}\text { Epipinoresinol, }(1 R, 2 S, 5 R, 6 R)-5^{\prime} \text {-O-Methylpluviatilol, } l \text {-Sesamin, } \\
l \text {-Asarinin }\end{array}$ \\
\hline & $N$-Alkylamide (5) & $\begin{array}{l}\text { Spilanthol, } N \text {-Isobutyl-2E,4E, } 8 Z, 10 Z \text {-dodecatetraenamide, } N \text {-Isobutyl- } \\
2 E, 4 E, 8 Z, 10 E \text {-dodecatetraenamide, } N \text {-Isobutyl-2,4,8-dodecatrienamide, } \\
N \text {-Isobutyl-2,4-dodecadienamide }\end{array}$ \\
\hline \multirow{3}{*}{ Sedative (5) } & Monoterpene (3) & Myrcene, Limonene, Eucalyptol \\
\hline & Phenylpropanoid (1) & Methyleugenol \\
\hline & Benzene derivative (1) & 3,5-Dimethoxytoluene \\
\hline \multirow{2}{*}{$\begin{array}{l}\text { Anti-spasmodic } \\
\quad \text { (9) }\end{array}$} & Monoterpene (7) & $\begin{array}{l}\text { Camphor, } l \text {-Borneol, } \beta \text {-Pinene, } \alpha \text {-Phellandrene, Eucalyptol, Terpinolene, } \\
\alpha \text {-Terpineol }\end{array}$ \\
\hline & Phenylpropanoid (2) & Methyleugenol, Estragole \\
\hline \multirow{4}{*}{$\begin{array}{l}\text { Anti-allergic } \\
\text { (8) }\end{array}$} & Monoterpene (2) & Limonene, $l$-Borneol \\
\hline & Phenylpropanoid (2) & Methyleugenol, Elemicin \\
\hline & Lignan (3) & Xanthoxylol, $l$-Sesamin, $l$-Asarinin \\
\hline & $N$-alkylamide (1) & $N$-Isobutyl-2E,4E,8Z,10E-dodecatetraenamide \\
\hline \multirow{2}{*}{$\begin{array}{l}\text { Cardiovascular } \\
\qquad(14)\end{array}$} & Monoterpene (9) & $\begin{array}{l}\alpha \text {-Pinene, } \beta \text {-Pinene, Terpinen-4-ol, } \alpha \text {-Terpineol, Eucalyptol, Camphor, } \\
l \text {-Borneol, Limonene, Terpinolene }\end{array}$ \\
\hline & Phenylpropanoid (5) & Methyleugenol, Elemicin, Kakuol, Hydroferulic Acid, Estragole \\
\hline \multirow{3}{*}{$\begin{array}{l}\text { Antitussive } \\
\quad(8)\end{array}$} & Monoterpene (4) & $\alpha$-Pinene, Camphor, Eucalyptol, Terpinen-4-ol \\
\hline & Phenylpropanoid (1) & Kakuol \\
\hline & $N$-alkylamide (3) & $\begin{array}{l}N \text {-Isobutyl-2E,4E,8Z,10Z-dodecatetraenamide, } N \text {-Isobutyl-2E,4E,8Z,10E- } \\
\text { dodecatetraenamide, } N \text {-Isobutyl-2,4,8,10,12-tetradecapentaenamide }\end{array}$ \\
\hline \multirow{2}{*}{$\begin{array}{l}\text { Hypothermic } \\
(2)\end{array}$} & Monoterpene (1) & Limonene \\
\hline & Phenylpropanoid (1) & Methyleugenol \\
\hline \multirow{2}{*}{$\begin{array}{l}\text { Anticonvulsant } \\
\text { (5) }\end{array}$} & Monoterpene (4) & Myrcene, Limonene, Terpinen-4-ol, $\alpha$-Terpineol \\
\hline & Phenylpropanoid (1) & Methyleugenol \\
\hline
\end{tabular}


Furthermore, among them (Table 5), 14 absorbed constituents possess analgesic activities, 27 absorbed constituents induce anti-inflammatory effects, five absorbed constituents exhibit sedative prosperities, nine absorbed constituents have antispasmodic actions, eight absorbed constituents have anti-allergic effects, 14 absorbed constituents bring cardiovascular benefits, eight absorbed constituents act antitussive activities, two absorbed constituents show hypothermal actions and five absorbed constituents possess anticonvulsant effects. These indicated that many different constituents of AR might act at the same target where the additive concentrations of them could reach an effective level for disease treatment. This inspire us to make a hypothesis on the mechanism of additive effect of multiple constituents of TCMs, which is very worthy of further research in the future and the research evidence may provide us an explanation of why does a traditional Chinese drug still have effective actions although the concentrations of its constituents are generally very low in blood.

\section{Experimental}

\subsection{Reagents and Materials}

Asari Radix et Rhizoma (AR) was purchased from Anguo Herb Market (Anguo City, Hebei Province, China). The sample was identified as the roots and rhizomes of Asarum heterotropoides Fr. Schmidt var. mandshuricum (Maxim.) Kitag. by one of the authors, Professor Shao-Qing Cai, and its voucher specimen (No. 6650) was deposited in the Herbarium of Pharmacognosy, School of Pharmaceutical Sciences, Peking University (Beijing, China).

Methyleugenol (Lot: PH3YH-MG), eucarvone (Lot: F1101-DLFG) and l-borneol (Lot: EPH8L-QQ) were obtained from TCI (Tokyo, Japan), 3,4,5-trimethoxytoluene (Lot: 19923) from Aladdin Industrial Inc. (Shanghai, China), and 3,5-dimethoxytoluene (Lot: 10099004) from Alfa Aesar (Heysham, UK). Safrole, kakuol, kakuol methyl ether, epipinoresinol, xanthoxylol and $l$-asarinin were separated from Asari Radix et Rhizoma, and 2,3,5-trimethoxytoluene was synthesized by the authors. The structures were confirmed by MS and NMR, and their purities were over 98\% (HPLC, area normalization method). $n$-Alkane mixture (C8-C20, Lot: 0001443129) was acquired from Fluka (Buchs, Switzerland) to calculate the retention indices (RI) of volatile constituents. Acetonitrile (HPLC grade) was purchased from Fisher (Fair Lawn, NJ, USA) and formic acid (HPLC grade) from Mreda Technology (Beijing, China). Sodium chloride was supplied by Sinopharm Chemical Reagent Co, Ltd (Beijing, China). Ethyl acetate (EtOAc) and methanol of analytical grade were obtained from Beijing Chemical Works (Beijing, China). Deionized water was prepared using a Milli-Q water purification system (Millipore, Billerica, MA, USA). The SPME fiber coated with $65 \mu \mathrm{m}$ polydimethylsiloxane/divinylbenzene (PDMS/DVB) was purchased from Supelco (Bellefonte, PA, USA).

\subsection{Preparation of AR EtOAc Extract}

Fifty g of AR was ground and passed through a 40 mesh sieve (mesh size $0.425 \mathrm{~mm}$ ) and then transferred into a $2000 \mathrm{~mL}$ flask containing $600 \mathrm{~mL}$ EtOAc. The flask was then sealed with a glass cap and placed in an ultrasonic bath at room temperature for $1 \mathrm{~h}$ for extraction. The extract was filtered and the filtrate was evaporated at $30{ }^{\circ} \mathrm{C}$ under vacuum using a Heidolph Laborota 4001 rotatory evaporator 
(Heidolph Instruments GmbH \& Co., Schwabach, Germany) until no solvent was distilled, yielding $1.2 \mathrm{~mL}$ (1.26 g) oily AR EtOAc extract.

\subsection{Animals and Sample Collection}

The animal studies protocol was approved by the Biomedical Ethical Committee of Peking University (Approval No. LA2011-76). Male albino rabbits weighing $2.5 \pm 0.1 \mathrm{~kg}$ were obtained from the Experimental Animal Center of Peking University Health Science Center (Beijing, China). 15 rabbits were randomly divided into three groups (five animals each): the AR EtOAc extract group (group b), the AR powder group (group c) and the blank group (group a).

The rabbits in the AR EtOAc extract group were administrated intranasally with a single dose of $50 \mu \mathrm{L}$ AR EtOAc extract in each nostril using a microlitre syringe attached with polyethylene tube $(2 \mathrm{~mm}$ in diameter). For intranasal administration of AR powder, the ground AR powder was passed through an 80 mesh sieve (mesh size $0.180 \mathrm{~mm}$ ) according to the instruction for nasal powder of the Chinese Pharmacopeia. The powder was weighed and placed in a $1 \mathrm{~mL}$ pipette tip connected to a rubber suction bulb. The pipette tip was then inserted into the animal nostril and $0.05 \mathrm{~g}$ AR powder was blown into each nostril. The rabbits in the AR powder group received the dosing twice a day for 10 days $(0.08 \mathrm{~g} / \mathrm{kg}$ per day) before the blood and the CSF samples were collected. The rabbits in the blank group were administrated intranasally with deionized water. During the administration, all animals were held in a supine position and kept in this position for $1 \mathrm{~min}$ after drug administration.

Blood samples were withdrawn in heparinized vacuum glass tubes via cardiac puncture, and then the samples of each group were combined into one sample. The plasma was separated by centrifugation at $3000 \mathrm{rpm}$ for $15 \mathrm{~min}$. CSF samples were obtained by cisternal puncture using a $1 \mathrm{~mL}$ syringe with 30 gauge needle, and the samples of each group were combined into one sample. The samples of rabbits in the AR EtOAc extract group were collected at 15 min after administration, which was the time point that the highest nociception inhibition of stimulus was observed according to our previous pharmacological experiment. The samples of rabbits in the AR powder group were taken at the 10th day after administration. All samples were collected while the rabbits were under anesthesia, and the samples were pretreated and analyzed within $24 \mathrm{~h}$.

\subsection{Automated HS-SPME-GC-MS Analysis}

A Shimadzu (Kyoto, Japan) GC/MS QP-2010 Ultra system equipped with an AOC-5000 autosampler was used for the automated analyses. Before use, SPME fibers were conditioned in accordance with the manufacturer's recommendations and for each fiber blank desorption was performed. $500 \mu \mathrm{L}$ plasma or CSF was placed in a $10 \mathrm{~mL}$ magnetic cap headspace vial with silicone/PTFE septum and $0.10 \mathrm{~g}$ sodium chloride was added. The PDMS/DVB fiber was then exposed to the headspace at $70{ }^{\circ} \mathrm{C}$ for $40 \mathrm{~min}$ while agitating. After this, the fiber was withdrawn into the needle and desorbed at $250{ }^{\circ} \mathrm{C}$ for 3 min into the GC injection port.

Chromatographic separations were carried out on a Restek Rxi-5MS $(30 \mathrm{~m} \times 0.25 \mathrm{~mm}, 0.25 \mu \mathrm{m}$ film thickness) capillary column (Bellefonte, PA, USA) using high-purity helium as the carrier gas at a flow rate of $1.2 \mathrm{~mL} / \mathrm{min}$. The temperature program was as follows: $80{ }^{\circ} \mathrm{C}$ (hold $3 \mathrm{~min}$ ), $20{ }^{\circ} \mathrm{C} / \mathrm{min}$ to $120{ }^{\circ} \mathrm{C}, 10{ }^{\circ} \mathrm{C} / \min$ to $140{ }^{\circ} \mathrm{C}, 20{ }^{\circ} \mathrm{C} / \min$ to $180{ }^{\circ} \mathrm{C}, 10{ }^{\circ} \mathrm{C} / \min$ to $210{ }^{\circ} \mathrm{C}$, then $30{ }^{\circ} \mathrm{C} / \mathrm{min}$ to $260{ }^{\circ} \mathrm{C}$ 
(hold $5 \mathrm{~min}$ ). The injection temperature was set at $250{ }^{\circ} \mathrm{C}$, and the split mode (split ratio 5:1) was used for the analysis of plasma and CSF from the AR EtOAc extract group whereas the splitless mode was used for that from the AR powder group. The spectrometers were operated in the electron-impact (EI) mode with electron energy of $70 \mathrm{eV}$ and source temperature of $200{ }^{\circ} \mathrm{C}$. The scan range was $45-450 \mathrm{amu}$, and the scan rate was $0.30 \mathrm{~s}$ per scan. The interface temperature was $260{ }^{\circ} \mathrm{C}$.

Identification of the absorbed constituents was based on comparison of their mass spectral data with those from NIST 08 library and authentic standards, along with their retention index (RI) calculated using the $n$-alkanes mixture [12].

\subsection{HPLC-APCI-IT-TOF-MS Analysis}

The HPLC-APCI-IT-TOF-MS ${ }^{\mathrm{n}}$ analyses were performed on a Shimadzu LCMS-IT-TOF system comprised of two LC-20AD pumps, an SIL-20AC autosampler, a CTO-20A column oven, an SPD-M20A PDA detector, a CBM-20A system controller, an APCI ion source and an IT-TOF mass spectrometer.

For pretreatment of samples, $5 \mathrm{~mL}$ plasma or $2.5 \mathrm{~mL}$ CSF was added with $10 \mathrm{~mL}$ or $5 \mathrm{~mL}$ EtOAc respectively, and then vortexed for $2 \mathrm{~min}$. The mixtures were centrifuged at $3000 \mathrm{rpm}$ for $15 \mathrm{~min}$, and the upper organic layer was transferred to a clean tube. The mixture was again extracted with the same volume of EtOAc followed by vortex mixing and centrifugation as described above. The upper layer from the two extractions were combined and evaporated to dryness at room temperature by nitrogen. The residue was then dissolved in $100 \mu \mathrm{L}$ methanol and $5 \mu \mathrm{L}$ was injected into the HPLC.

Chromatographic separations were achieved at $30{ }^{\circ} \mathrm{C}$ with a Phenomenex Gemini-NX $\mathrm{C}_{18}$ column (5 $\mu \mathrm{m}$ particle size, $250 \times 4.6 \mathrm{~mm})$, using water-formic acid $(100: 0.1, v / v)(\mathrm{A})$ and acetonitrile (B) as the mobile phase. The mobile phase was delivered at a rate of $1.0 \mathrm{~mL} / \mathrm{min}$ and the gradient program was as follows: $11 \%-42 \% \mathrm{~B}$ at $0-30 \mathrm{~min}, 42 \%-45 \% \mathrm{~B}$ at $30-45 \mathrm{~min}, 45 \%-54 \% \mathrm{~B}$ at $45-48 \mathrm{~min}$, $54 \%-58 \%$ B at $48-55 \mathrm{~min}, 58 \%-60 \%$ B at $55-60 \mathrm{~min}, 60 \%-90 \%$ B at $60-65 \mathrm{~min}$ and $90 \%-100 \%$ B at $65-75 \mathrm{~min}$. After the elution, $100 \% \mathrm{~B}$ was kept for $10 \mathrm{~min}$ to flush the column. For mass detection, the mass spectrometer was programmed to carry out a full scan over $m / z 100-1000\left(\mathrm{MS}^{1}\right)$ and $\mathrm{m} / \mathrm{z}$ 50-1000 $\left(\mathrm{MS}^{2}\right.$ and $\left.\mathrm{MS}^{3}\right)$ in positive ion detection mode. Nitrogen was used as the nebulizing gas and the flow rate was $2.0 \mathrm{~L} / \mathrm{min}$. The interface temperature, the curved desolvation line temperature and the heat block temperature was $400{ }^{\circ} \mathrm{C}, 250{ }^{\circ} \mathrm{C}$ and $200{ }^{\circ} \mathrm{C}$, respectively. The detector voltage was $1.70 \mathrm{kV}$, and the drying gas pressure was $40.0 \mathrm{kPa}$. The data were processed by Shimadzu software (LCMSsolution Version 3.60, Formula Predictor Version 1.2 and AccurateMass Calculator).

\section{Conclusions}

In this paper, HS-SPME-GC-MS and HPLC-APCI-IT-TOF-MS ${ }^{\mathrm{n}}$ were used for the first time to identify the volatile and non-volatile absorbed constituents in rabbits after intranasal administration of AR EtOAc extract and AR powder. For the first time, 46 absorbed constituents of AR containing 13 monoterpenes, 10 phenylpropanoids, four benzene derivatives, two alkanes, nine $N$-alkylamides and eight lignans were found could be absorbed into the rabbit plasma, whereas 28 absorbed constituents containing 14 monoterpenes, five phenylpropanoids, four benzene derivatives, two alkanes, two $\mathrm{N}$-alkylamides and one lignan could be absorbed into the CSF. Among these absorbed constituents, 46 constituents were detected in the plasma and 28 constituents were found in the CSF after intranasal 
administration of AR EtOAc extract (Table 4), and 10 constituents were identified in the plasma and 6 constituents were detected in the CSF after intranasal administration of AR powder. These indicated that many types of constituents of TCM can be simultaneously absorbed at the nasal cavity into both rabbit blood and CSF. Through bibliography retrieval, the bioactivities of 33 absorbed constituents were found to be related to the pharmacological actions of AR (detailed information are listed in Table 5 and Supplemental Materials Table S1).

Furthermore, we have done several pharmacological experiments on AR EtOAc extract and its absorbed constituents. We find that AR EtOAc extract intranasally administrated exerts its analgesic effect more quickly and more potent than that of oral administration, and the four major absorbed constituents of AR (3,5-dimethoxytoluene, 2,3,5-trimethoxytoluene, 3,4,5-trimethoxytoluene, methyleugenol) and their combinations have significant analgesic effect and anti-inflammatory effect. We are applying for a Chinese patent based on these findings and for this reason the pharmacological results will be published elsewhere in the future.

These findings will enhance our understanding of the effective substances of AR, and this paper can be regarded as a paradigm for the systematic screening and identification of absorbed constituents of TCMs used in nasal therapy. Based on our results and literature retrieval, we make a hypothesis on the mechanism of additive effect of multiple constituents of TCMs, which is very worthy of further study and verification in the future.

\section{Supplementary Materials}

Supplementary materials can be accessed at: http://www.mdpi.com/1420-3049/19/4/4857/s1.

\section{Acknowledgments}

This study was financially supported by General Program of National Natural Science Foundation of China (Grant No. 81274073).

\section{Author Contributions}

Participated in research design: Feng $\mathrm{Xu}$, Ming-Ying Shang, Xuan Wang and Shao-Qing Cai. Conducted experiments: Li Chen and Feng Xu. Contributed new reagents or analytic tools: Yu Jing and Guang-Xue Liu. Performed data analysis: Li Chen and Feng Xu. Wrote or contributed to the writing of the manuscript: Li Chen, Feng Xu, De-Mei Xie, Xuan Wang and Shao-Qing Cai. Other: Shao-Qing Cai acquired funding for the research.

\section{Conflicts of Interest}

The authors declare no conflict of interest.

\section{References}

1. Gao, S.Z. Zhongyi Biliaofa Quanshu A Comprehensive Treatise on Nasal Therapy of Traditional Chinese Medicine, 1st ed.; Huaxia Publishing House: Beijing, China, 1994; pp. 33-401. 
2. Pires, A.; Fortuna, A.; Alves, G.; Falcão, A. Intranasal drug delivery: How, why and what for? J. Pharm. Pharm. Sci. 2009, 12, 288-311.

3. Yu, L.; Chen, Q.L.; Guo, S.S.; Liu, Y.D. Overview on intranasal delivery of Chinese medicine in curing systemic diseases. Asia Pac. Trad. Med. 2008, 4, 88-91.

4. Feng, J.; Li, F.; Zhao, Y.; Feng, Y.; Abe, Y. Brain pharmacokinetics of tetramethylpyrazine after intranasal and intravenous administration in awake rats. Int. J. Pharm. 2009, 375, 55-60.

5. Yao, Z.; Lu, Y.; Du, S.; Chen, X.; Wang, Y. Study on pharmacokinetics of jasminoidin in rabbits administered Xingnaojing Naristillae by nasal medication. Chin. J. Chin. Mater. Med. 2010, 35, $1871-1873$.

6. Gong, Z.N.; Xu, L.Y.; Song, J.Z.; Tao, J.S.; Ma, S.R. Study on rat nasal administration with emulsion of Chinese herb Angelica Dahurica. Chin. J. Clin. Pharm. 2001, 10, 370-373.

7. Guo, X.J.; Li, J.Y.; Lin, C.Y.; Yang, F. Exploration on nasal administration of traditional Chinese medicine for treating brain diseases. J. Guangdong Pharm. Coll. 2011, 27, 211-214.

8. Zhao, B.C.; Wu, H.Y. Study on the prescribing rules of traditional Chinese medicines for the treatment of migraine by intranasal administration. Asia Pac. Trad. Med. 2010, 6, 132-133.

9. Han, A.R.; Kim, H.J.; Shin, M.; Hong, M.; Kim, Y.S.; Bae, H. Constituents of Asarum sieboldii with inhibitory activity on lipopolysaccharide (LPS)-induced NO production in BV-2 microglial cells. Chem. Biodivers. 2008, 5, 346-351.

10. Wagner, H.; Bauer, R.; Melchart, D.; Xiao, P.G.; Staudinger, A. Chromatographic Fingerprint Analysis of Herbal Medicines, 2nd ed.; Springer: Vienna, Austria, 2011; Volume 1, pp. 45-58.

11. Yuan, X.Q.; Sun L.F.; Zheng, J. Analgesic components of Asarum and its mechanisms. Shanghai J. Trad. Chin. Med. 2009, 43, 72-75.

12. Li, C.; Xu, F.; Cao, C.; Shang, M.Y.; Zhang, C.Y.; Yu, J.; Liu, G.X.; Wang, X.; Cai, S.Q. Comparative analysis of two species of Asari Radix et Rhizoma by electronic nose, headspace GC-MS and chemometrics. J. Pharm. Biomed. Anal. 2013, 85, 231-238.

13. Yan, G.; Li, Q.; Tan, H.; Ge, T. Electrospray ionization ion-trap time-of-flight tandem mass spectrometry of two furofurans: sesamin and gmelinol. Rapid Commun. Mass Spectrom. 2007, 21, 3613-3620.

14. Struijs, K.; Vincken, J.P.; Gruppen, H. Comparison of atmospheric pressure chemical ionization and electrospray ionization mass spectrometry for the detection of lignans from sesame seeds. Rapid Commun. Mass Spectrom. 2008, 22, 3615-3623.

15. Eklund, P.C.; Backman, M.J.; Kronberg, L.A.; Smeds, A.I.; Sjöholm, R.E. Identification of lignans by liquid chromatography-electrospray ionization ion-trap mass spectrometry. J. Mass Spectrom. 2008, 43, 97-107.

16. Xing, J.; Zhang, S.Q.; Zhong, D.F.; Jia, J.M. Rapid identification of Acanthopanax senticosus mixture by liquid chromatography-electrospray ion trap mass spectrometry. J. Chin. Mass Spectrom. Soc. 2004, 25, 198-203.

17. Quang, T.H.; Ngan, N.T.; Minh, C.V.; Kiem, P.V.; Tai, B.H.; Thao, N.P.; Song, S.B.; Kim, Y.H. Anti-inflammatory and PPAR transactivational effects of secondary metabolites from the roots of Asarum sieboldii. Bioorg. Med. Chem. Lett. 2012, 22, 2527-2533. 
18. Boonen, J.; Baert, B.; Burvenich, C.; Blondeel, P.; de Saeger, S.; de Spiegeleer, B. LC-MS profiling of $\mathrm{N}$-alkylamides in Spilanthes acmella extract and the transmucosal behaviour of its main bio-active spilanthol. J. Pharm. Biomed. Anal. 2010, 53, 243-249.

19. Thomsen, M.O.; Fretté, X.C.; Christensen, K.B.; Christensen, L.P.; Grevsen, K. Seasonal variations in the concentrations of lipophilic compounds and phenolic acids in the roots of Echinacea purpurea and Echinacea pallida. J. Agric. Food Chem. 2012, 60, 12131-12141.

20. Mudge, E.; Lopes-Lutz, D.; Brown, P.; Schieber, A. Analysis of alkylamides in Echinacea plant materials and dietary supplements by ultrafast liquid chromatography with diode array and mass spectrometric detection. J. Agric. Food Chem. 2011, 59, 8086-8094.

21. Cech, N.B.; Eleazer, M.S.; Shoffner, L.T.; Crosswhite, M.R.; Davis, A.C.; Mortenson, A.M. High performance liquid chromatography/electrospray ionization mass spectrometry for simultaneous analysis of alkamides and caffeic acid derivatives from Echinacea purpurea extracts. J. Chromatogr. A 2006, 1103, 219-228.

22. Yasuda, I.; Takeya, K.; Itokawa, H. Structures of Amides from Asiasarum heterotropoides MAEK. var. mand shuricum MAEK. Chem. Pharm. Bull. 1981, 29, 564-566.

23. Zhang, S.X.; Tani, T.; Yamaji, S.; Gao, X.L.; Wang, X.; Cai, S.Q.; Zhao, Y.Y. Studies on chemical constituents from radix and rhizome of Asarum longerhizomatosum. Chin. Trad. Herba. Drugs 2002, 33, 297-299.

24. Illum, L. Nasal drug delivery-Possibilities, problems and solutions. J. Control. Release 2003, 87, 187-198.

Sample Availability: Samples of the compounds G11, G12, G17, G18, G20 (L6), G21, G22 (L7), L5, L9, L10, L11, and L15 are available from the authors.

(C) 2014 by the authors; licensee MDPI, Basel, Switzerland. This article is an open access article distributed under the terms and conditions of the Creative Commons Attribution license (http://creativecommons.org/licenses/by/3.0/). 